\title{
MINIMUM WATER CONSUMPTION METHOD SCREENING OF VELVET BEAN (MUCUNA SP.) PROCESSINGS TO PRODUCE FUNCTIONAL FOOD INGREDIENTS
}

\begin{abstract}
Velvet bean (Mucuna sp.) has been proven containing many beneficial compounds that can be implemented in pharmaceutical and medicines but less noticed for functional foods even though traditionally it is consumed as daily foods or snacks. The indigenous food preparation such as velvet bean Tempe warrants scientific investigation to help society with better public health management. The objective of the review is to select the best method for functional food ingredient product development using velvet beans and provide hypothetical health-oriented food processing e.g. velvet bean flour as functional food ingredients with a focus on less water consumption during processing. Steaming is the selected method.
\end{abstract}

Keywords: Food ingredient, functional food, lower water use processing, safe velvet bean

\begin{abstract}
ABSTRAK
Koro benguk (Mucuna sp.) telah terbukti memiliki banyak senyawa yang menguntungkan yang dapat diterapkan dalam farmasetika dan pengobatan tetapi kurang diperhatikan untuk pangan fungsional meskipun secara tradisional dikonsumsi sebagai pangan sehari-hari atau camilan. Penyiapan pangan secara asli seperti tempe koro benguk memerlukan investigasi ilmiah untuk membantu masyarakat dengan pengelolaan kesehatan masyarakat yang lebih baik. Tujuan dari review ini adalah untuk menyeleksi metode terbaik untuk pengembangan ingridien pangan fungsional menggunakan koro benguk dan memberikan hipotesa pengolahan pangan yang berorientasi kesehatan, misalnya, tepung koro benguk sebagai ingredien pangan fungsional dengan fokus pengolahan yang sedikit memerlukan air. Pengukusan sebagai metode yang dipilih.
\end{abstract}

Kata kunci: Ingredien instan, koro benguk yang aman, pengolahan dengan sedikit air, pangan fungsional

\section{Article Information}

Article Type: Review Paper Journal Type: Open Access Volume: 2 Issue 1

Manuscript ID v2n1342-1

Received Date 10 April 2020

Accepted Date 23 July 2020

Published Date 31 August 2020

DOI:

10.33555/jffn.v2i1.34

Corresponding author: Indah Epriliati

Surabaya, Indonesia, 610265

Email:

margarethaiev@gmail.com

Citation:

Epriliati, I. 2020. Velvet bean (mucuna Sp.) processings to produce functional food. $\mathrm{J}$. Functional Food \& Nutraceutical, 2(1), pp.1-28.

Copyright: $\odot 2020$ Swiss German University. This is an open-access article distributed under the terms of the Creative Commons Attribution 4.0 Internationa License, which permits unrestricted use, distribution and reproduction in any medium, provided the original author and source are credited. 


\section{INTRODUCTION}

Velvet beans, Mucuna genus, has recently become important and it seems progressively being explored for potential foods with beneficial health effects so the genus members are potential functional food resources. It has almost been four decades the intensive research on Mucuna $s p$. revealing various aspects, ultimately those of nutritional and health effects, antinutrients, and processing types. The abundant data yet is awaiting to be optimally used to support sustainable healthy living and to fixing the degrading agricultural system for livelihood. Velvet bean is a well-known covering crop to replenish humus and nitrogen fixation as green manures in the farming area. Moreover, mucuna showed the capability to extract $>30 \%$ of soil hydrocarbon into leaf (Nwaichi et al., 2009) and recommended as decontaminator plant to tackle oil spills in the land (Eucharia and Edward, 2010). Although L-DOPA is released into the soil as a weed controller by mucuna affecting the growth of other plants, its allelochemical effects lose after 12 weeks postharvest period tested using S. stenocarpa (Eucharia and Edward, 2010).

There are two velvet bean climbing characteristics, i.e. woody and herbaceous; totally, it is comprised of around 105 species in the world either growing in tropical or subtropical regions (Ingalhalikar et $a l ., 2017)$. The woody velvet bean is more likely to be newcomers whose different types of pods, seed shapes, and general floral colors. M. laticifera, $M$. macrocarpa, and M. birdwoodiana are those belong to woody velvet bean whereas herbaceous velvet bean are $M$. pruriens, M. utilis, and many others which have been explored earlier for foods and medicines. Hence, Mucuna sp. truly still becomes interesting research subjects besides the abundant research already obtained since the 1970s. Historically, one of the ancient documents about velvet bean in Indonesia was pessimistic about the possibility of good uses of velvet bean along with human's experiences in interactions with velvet bean (Heyne, 1987) because at that moment (Colonialism) they mostly used velvet bean plant for green manures in the farms while the leaves and seeds were consumed or used as part of traditional medicine by native population yet lack of scientific data. Therefore, various experiments were carried out starting from green manure, in vivo for cattle feeds to scientific investigation on traditional medicines. But, now velvet bean has obtained much attention for its previously negative effects on human life through a new concept of functional food or health-oriented food. The compounds responsible for various symptoms such as vomiting, headache, or itchiness have been revealed in studies in many countries.

Indigenous processing methods are the best resources to screen the goals of this research. Vadivel and Bielsaski (2012) evaluate indigenous processing of velvet bean from India for velvet beans collected from around the world (Mexico, India, USA, Zimbabwe, Nigeria, Benin, Guatemala, Ghana, Kenya, and Guinea) and found such processing is safe to be applied continuously as food base products at the household level, not as drugs. Nowadays, in Indonesia velvet bean varieties are still cultivated generally in suboptimal areas where water and/or clean water scarce. The ancients documents indicate velvet bean species found in Indonesia namely: (a) $M$. diabolica Backer, (b) M. junghuhniana Backer (M. blumei Burck), (c) M. pruriens DC (M. prurita Hook.), (d) Spatholobus ferrugineus Benth, (e) Spatholobus littoralis Hassk., (f) Calopogonium mucunoides Desv., and (g) M. bakeri KDS (Heyne, 1987). This is still apparent to be cultivated.

Nevertheless, until now velvet bean is underutilized crops due to its antinutrients, toxin and itching characteristics of its hairs. Antinutrients in velvet bean include tannin, digestibility enzymic inhibitors (antitrypsin, antiamilase), phytates, and antihemagglutinin. Based on the names, it describes what kind of crops velvet bean is. For instance, devil bean, pruriens which is in Latin referring to "itching sensation" from the fine hairs (Sridhar and Bhat, 2007) covering velvet bean pods. The pods have hairs containing 5hydroxytryptamine (serotonin) and mucunain (a protein) that cause severe itches (erythema) (Giuliano and Allard, 2001). Yet, behind the negative traits, velvet bean suddenly wakes the world up from its contributions for managing health, venoms, malnutrition for marginal people after Ayurvedic messages revealed. Velvet bean's 
uniqueness that grows in arid areas, making it is blessed with so many bullets to survive in such poor soils. In contrast to its comparable nutrition to soybean, antinutrients and alkaloids, cyanides and a non-protein amino acid seem complementarily required by velvet bean to facing a battle in environmental challenges. Velvet bean research, especially those herbaceous, started increasing since its content of L-DOPA (3,4-dihydroxy-Lphenylalanine) found to be capable of treating Parkinson's disease (Shaw and Bera, 1993; Prakash and Tewari, 1999; Pierson et al., 2004). Velvet bean exploration is re-born (Buckles, 1995) but in Indonesia, continuous research on velvet bean kept going on including (a) tempe of velvet bean which is continuously carried out in the Javanese society (Sardjono et al., 2012; Wanita and Rahayu, 2011), (b) plantation of velvet bean (Pramono, 2010), (c) food product development of velvet bean (Sardjono et al., 2012; Wanita and Rahayu, 2011; Sudiyono, 2010), and (d) Pharmacology of velvet beans (Sardjono, 1995; Winarni et al., 2011; Sardjono et al., 2018).

A systematic and comprehensive program has not been done for commercialization activities. It is time to transform the research results into an industrial system from agriculture to public health due to its potential safer protection for land from destructive inorganic fertilizers. It is demanded to enrich the soil with organic matters and selective herbicide to control weeds. Among them, the species of Mucuna pruriens as a cover crop widely accepted for enhancement of water infiltration into the soil, softening the soil, improvement of soil fertility and to suppress the weeds (Acanthospermum hispidum, Euphobia hirta, Senescio vulgaris, Oxygonum sinuatum, Schkuria pinnata, Richardia brasiliensis, Bidens pilosa, Sonchus oleraceae)(Osei-Bonsu et al. 1994; Mwangi et al., 2006). Interestingly, all antinutrients which are water-soluble simply leach out during soaking, rinsing, or washing. However, the arid or semiarid areas also mean that there is not much water available to control the antinutrients to the safe level doses during processing in such areas.

Although intensive research has been done on diverse species of mucuna from various points of view, there are still left problems required to be tackled. Hence, there is an urgent call to fix the food processing that saves nutrients and simultaneously reduces the antinutrients at safe levels but retains L-DOPA content at physiologically functional levels for neural health in the way that uses minimum consumption of water. And current society demands convenience food products so instant food product or food ingredients to support instantaneous characteristics is worthy to be investigated and implemented for sustainable agricultural-based livelihood.

The objectives of this review are (a) to screening from publications a candidate to be developed for minimum water consumption type processing of velvet beans including those studied from ancient documents about velvet bean in Indonesia; (b) to estimate the amount of water for each processing at the similar basis of velvet bean feeding quantity in the processing. Finally, it will recommend the processing type which requires less water for further investigations at scaling up to pilot plant for commercial functional food ingredient production in Indonesia.

\section{MUCUNA PLANT}

Taxonomically, velvet bean species belongs to the family of Fabaceae, some referring it with the name of Stizolobium (Sridhar and Bhat, 2007). Regarding its excellent chemical composition resembling soybean, velvet bean has been developed for combating malnutrition as well as curing particular diseases by usages of alkaloids in the seeds. The most popular species is $M$. pruriens which has several varieties, e.g. in Indonesia $M$. hirsuta W. and A., M. utilis Wall, M. velutina HASSK., $M$. capitata W. and A., and $M$. cochinchinensis A, Chev., M. nivea W. and A., Stizolobium niveum O.K. (Asia Nivea) as well as M. lyoni Merr (America lyoni) (Heyne, 1987). The varieties in Mucuna pruriens var. pruriens produce seeds richer in levodopa (Eucharia and Edward 2010) compared to others that can act as a direct precursor of the neurotransmitter dopamine and strongly affect sexual function (Giuliano and Allard, 2001). The characteristics of velvet seeds are described in Table 1. 
Tabel 1. Characteristics of mucuna seeds

\begin{tabular}{|c|c|c|c|c|}
\hline & Direct observation & Bhat et al. (2008) & Sardjono et al. (2012) & Heyne (1987) \\
\hline origin & Yogyakarta, Indonesia & & Yogyakarta, Indonesia & $\begin{array}{l}\text { Sumatera, Bali, Java, Molucas, Madura and } \\
\text { its small islands, Celebes islands }\end{array}$ \\
\hline shape & round-flat & flat or varied & oval and flat & $\begin{array}{l}\text { flat, a little bigger than other legumes; very } \\
\text { glossy (M. hirsuta W. and A.) }\end{array}$ \\
\hline color & $\begin{array}{l}\text {-grey } \\
\text {-yellowish-brown } \\
\text {-white } \\
\text {-black } \\
\text {-white grey spotted } \\
\text {-black patterned } \\
\text {-brown patterned }\end{array}$ & black & $\begin{array}{l}\text { white, black, white with } \\
\text { black dots }\end{array}$ & $\begin{array}{l}\text { white from a purple flower (M. hirsuta W. } \\
\text { and A.); grey seed coat, white with spots, } \\
\text { yellowish pale brown background with } \\
\text { brown lines, deep black; big seed and } \\
\text { glossy, initially red then dark brown almost } \\
\text { black (M. capita W. and A.) }\end{array}$ \\
\hline weight (g/100 seeds) & & $43.61 \pm 1.06$ & $96.3^{*}$ & \\
\hline coat $(\mathrm{g})$ & & $0.07 \pm 0.01$ & & \\
\hline cotyledon $(\mathrm{g})$ & & $0.37 \pm 0.08$ & & \\
\hline length $(\mathrm{cm})$ & & $1.2 \pm 0.13$ & 1.491 & \\
\hline diameter $(\mathrm{cm})$ & & $0.78 \pm 0.12$ & 0.682 & \\
\hline thickness $(\mathrm{cm})$ & & $0.59 \pm 0.08$ & & \\
\hline hilum (cm) & & $0.44 \pm 0.03$ & & \\
\hline smell & specific velvet beans & & $\begin{array}{l}\text { characteristics of velvet } \\
\text { beans }\end{array}$ & \\
\hline taste & & & bitter & \\
\hline appearance & & & & $\begin{array}{l}\text { parts of plant is covered by fine golden or } \\
\text { brown hairs which causes itchiness, glossy } \\
\text { in the pods }\end{array}$ \\
\hline ethnobotany & & & & $\begin{array}{l}\text { many cases indicated headache, vomiting } \\
\text { and poor appetite when consumption of } \\
\text { velvet bean meals without soaking step; } \\
\text { proper processing gives better body weights } \\
\text { or good harvest }\end{array}$ \\
\hline
\end{tabular}

Indonesian sources of velvet bean germplasm is still underexplored and important notes from ancient documents strongly recommend waterintensive uses during food processing. Tempeh making from velvet seeds is still happening in Java in particularly remote areas. Figure 1 shows Mucuna sp. used as tempeh making in Yogyakarta, Indonesia. Exploration for sustainable agriculturalfood management in environmentally friendly ways can holistically keep balancing health food requirements and soil maintenance for sustainable agricultural practices.

\section{L-DOPA}

All species members of Mucuna sp. have been proven to contain L-DOPA either in seeds, leaves, and other parts of the plant. This compound is grouped into allelochemicals. L-DOPA is a nonprotein amino acid L-3,4dihydroxyphenylalanine. It is categorized as a product of biotransformation i.e. a single amino acid L-tyrosine is hydroxylated into L-DOPA by tyrosinase (EC 1.14.18.1) in a pathway of melanin biosynthesis (Raval et al., 2012). L-DOPA becomes an active biomolecule different from $\mathrm{L}$ tyrosine. In the velvet bean plant, it is a precursor of many alkaloids, catecholamines, and melanin syntheses and is released into soils, inhibiting the growth of nearby plant species acting as allelochemicals (Soares et al., 2014). As allelochemicals, L-DOPA controls soil and other plant growths (Rogers et al., 2004). L-DOPA resists attacks from insects and thus it can control biological infestation during storage too (Balogun and Olatidoye, 2012). Based on worldwide investigations (India, America, and Africa) on 3638 mucuna accessions regarding the L-DOPA in velvet eban plant Sridhar and Bhat (2007) concluded that : (1) black seeds contain higher LDOPA compared to white seeds (Vadivel and Janardhanan, 2000), (2) geographical location of cultivations affect L-DOPA contents, i.e. closer to the equator the seeds contain more L-DOPA (Lorenzetti et al., 1998), and (3) interaction of 
genotype and accession is stronger than genotype and geographic location but latitude could affect several accession more (Capo-chichi et al., 2003), furthermore, L-DOPA contents at the early stage of maturation is lower than that of fully mature seeds.

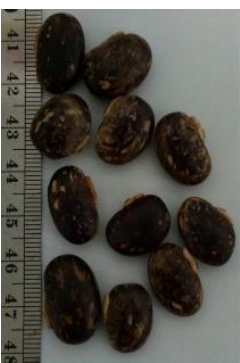

A

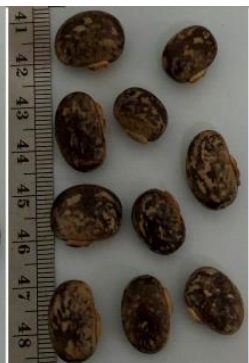

B

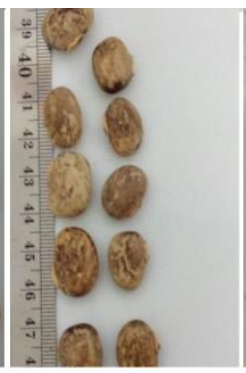

C

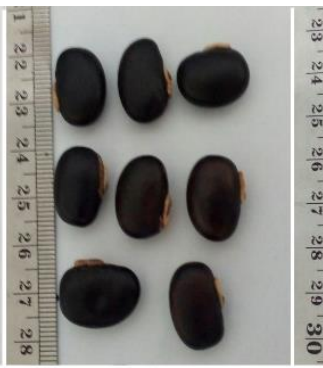

$E$

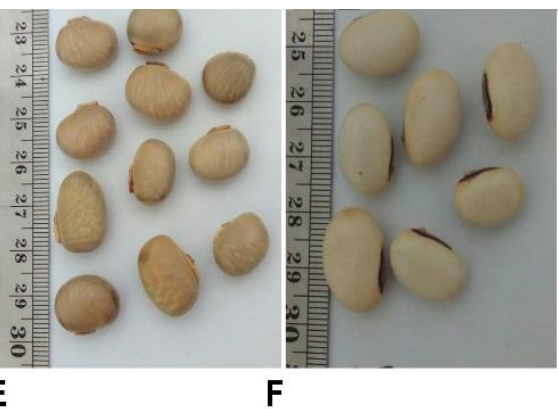

Figure 1. Mucuna pruriens currently direct observation found in Indonesia in 2018 (A-C mottled, (D) black, (E) Kaunch, and (F) white.

L-DOPA more likely decreases during the germination of velvet seeds because it is used as $\mathrm{N}$ supply or preparing soil where it is starting to grow. During its growth velvet bean releases LDOPA into soils and it affects other plants around it as an allelochemical. Then the L-DOPA diffuses out spreading wider due to rains (Ferreira and Janick, 2004) and watering activity thus no weed growths in the area around it. Meanwhile, the allelochemical DOPA can affect other plants through growth suppression decreasing dry matter yields (for $S$. stenocarpa $1.58 \mathrm{~g}$ dry matters obtained in control but it became $1.06 \mathrm{~g}$ when grows mixed with $M$. pruriens var. pruriens), smaller leaf areas, and poor growth rates but not affecting the capacity to germinate (Eucharia and Edward, 2010). L-DOPA suppresses radicle growth but less attacks hypocotyl growth and may ineffectively affect germination (Fujii, 2003). Towards soybean, cucumber, and maize L-DOPA affect its lignin related synthesis in the cell wall because it is effectively absorbed resulting in increased tyrosine, lignin biomarkers, and phenylalanine but its root growth is inhibited. It is categorized as a strong allelochemical with $\mathrm{EC}_{50}$ ranges from $5-50 \mathrm{mg} / \mathrm{mL}$. Its herbicidal effects at 1,500 and 3,000 ppm affects wild mustard (Sinapsis arvense), creeping thistle (Cirsium arvense), filed poppy (Papaver rhoeas), and henbit (Lamium amplexicaule), but it does not significantly affect wheat and barley through inhibiting root growth (Topa and Kocacaliskan, 2006).

Homeostasis of iron and amino acid metabolism in mammals is also affected by L-DOPA related to ion transporter encoding (Golisz et al., 2011). LDOPA is converted into dopamine in the brain and body by enzyme L-aromatic amino acid decarboxylase which directly controls the movement of the body (Raval et al., 2012). In patient of Parkinson disease L-DOPA was found to increase incorporation of L-DOPA-protein in lymphocyte cell proteins as a "toxic" situation (Rogers et al., 2004).

Pivotal effects of dose-dependent L-DOPA and its metabolite dopamine on neural-tissues can be described that besides alleviating the disease symptoms, L-DOPA may contribute to disease progression. Acute side effects of L-DOPA, which include nausea, vomiting, and orthostatic hypotension (dizziness), are correlated with plasma levels. It is important to note that dopamine does not pass the blood-brain barrier in sufficient quantities, thus only a small percentage of $\mathrm{L}$ DOPA reaches the brain after systemic administration. Moreover, L-DOPA is quickly metabolized peripherally, therefore high systemic L-DOPA doses are required to achieve the clinical effect (3-4 g of L-DOPA/day). Because immediate 
side-effects are directly related to L-DOPA peak plasma levels, L-DOPA was, in recent years, administered in combination therapy with other compounds such as decarboxylase and COMT (Catechol-amine-O-methyl-transferase) inhibitors to prevent peripheral metabolism. To prevent the metabolism of dopamine in the brain, MAO (Mono-amine oxidase) inhibitors were also used. With these additives, it was possible to reduce the daily required dose of L-DOPA to an average of about $600 \mathrm{mg} /$ day. However, these additives were only partially capable of reducing the toxic side effects of the treatment with levodopa and could not prevent disease progression.

In vitro synthesis of L-DOPA from amino acid tyrosine by soil isolate, Penicillium jensenii showed the production of alpha methyl DOPA along with L-DOPA. It is proposed that alpha methyl DOPA as a candidate to prevent unmetabolized L-DOPA from intensive L-DOPA metabolism for movement control after transformation into dopamine in the brain. Alpha methyl DOPA will be a delivery facility of unmetabolized L-DOPA so that the injured nerve cells have chances to recover instead of losing LDOPA for movement intensively (converted into dopamine) (Raval et al., 2012). It may indirectly control the toxic situation by L-DOPA-protein in lymphocyte cells. Fermentation would give a window opportunity to put biotransformation of tyrosine for both L-DOPA and alpha-methyl DOPA for a special goal towards functional foods containing L-DOPA mixed with alpha-methyl DOPA for the diet of Parkinson's sufferers of neural diseases.

\section{VELVET BEAN USES}

Velvet bean has intensively been revealed as one of functional food and simultaneously nutraceutical resources from ancient Ayurveda document in India. It is one of the potential pharmaceutical resources for neural related disease, proasidiac, snake bite venom, etc. The potential compounds highly important in pharmaceuticals include 1methyl-3-carboxy-6,7-dihydroxy-1 2,3,4tetrahydroisoquinolone, 5-hydroxytryptamine, 5methoxy-n,n-dimethyltryptamine-n-oxide, 5oxyindole-3-alkylamine, 6-methoxyharman,
Alanine, Arachidic-acid, Arginine, Aspartic-acid, Behenic-acid, Beta-carboline, Beta-sitosterol, Bufotenine, Choline, Cis-12,13-epoxyoctadectrans-9-cis-acid, Cis-12,13-epoxyoctadec-trans-9enoic-acid, Cystine, DOPA, Gallic-acid, Glutamicacid, Glutathione, Glycine, Histidine, L-DOPA, Lecithin, Leucine, Linoleic-acid, Mucunadine, Mucunain, Mucunine, Myristic-acid, N,ndimethyltryptamine, N,n-dimethyltryptamine-noxide, Nicotine, Oleic-acid, Palmitic-acid, Palmitoleic-acid, Phenyalanine, Phosphorus, Proline, Protein, Prurienidine, Prurienine, Saponins, Serine, Serotonin, Stearic-acid, Threonine, Tryptamine, Tyrosine, Valine, Vernolic-acid (Phytochemical and Ethnobotanical Databases at Phytochemical Database, USDAARS-NGRL, Beltsville Agricultural Research Center, Beltsville, Md).

The uses of velvet bean are not limited for general foods only because of famine, but also the intentional uses for particular goals regarding health and curing. Traditionally, velvet bean is used as a carminative, hypotensive, and hypoglycemic agent. Moreover, it is also used as anodyne, antidotal, aphrodisiac, diuretic, nervine, resolvent, rubefacient, and vermifuge; used for anasarca, asthma, cancer, cholera, cough, diarrhea, dog bite, dropsy, dysuria, insanity, mumps, pleuritis, ringworm, snakebite, sores, syphilis, tumors, and worms (Divya et al., 2017). Matured beans are consumed widely in Asia such as India, Sri Lanka, Ghana and Nigeria (Sridhar and Bhat 2007), Mozambique and Malawi (Infante et al., 1990; Gilbert, 2002) as well as in Indonesia and, Japan (Higasa et al., 1996) and they are considered as safe (Diallo et al., 2002) food products. These seeds are also reported to be rich in antioxidant properties (Tripathi and Upadhyay, 2001). LDOPA is also claimed to have a high antioxidant capacity (Raval et al., 2012; Balogun et al., 2017).

\section{NUTRITIONAL PROPERTIES OF VELVET BEAN}

The pulished nutritional analysis of velvet beans is presented in Table 3. It can be seen that various mucuna varieties would give relatively similar nutritional provisions for human needs: carbohydrates with a sufficient amount of fibers, 
proteins, high minerals (ash), and moderate levels of lipids. Any species of mucuna can be useful germplasm worldwide. Further detailed ash, fatty acids, and amino acid compositions listed in Table 4 - 6 strongly recommend balance nutrients required for any age groups and genders can be obtained from mucuna seed-based food products. Iron minerals exist in all mucuna species indicating that the beans can be good iron sources regardless of the antinutrients in them. The potassium contents are high thus it can be good contributions for diet components for people suffering from heart diseases and blood pressure. Calcium and phosphor with low sodium contents may a good composition for osteoporosis controls. Furthermore, micronutrient minerals such as zinc, cuprum, selenium, and mangan also provide better supply for people with diabetes mellitus problems. Fatty acid compositions indicate that the polyunsaturated-saturated ratios $>1$ would be less risk for health problems for instance people living with heart diseases. Many essential amino acids are available in velvet beans of different species; indeed, methionine is the least existing amino acid in them, thus diverse foods are recommended.

Table 2. Uses of velvet beans

\begin{tabular}{|c|c|c|}
\hline Velvet bean based food products & Locations & References \\
\hline velvet bean tempe-meal, dish & Indonesia & $\begin{array}{l}\text { Pramono (2010) } \\
\text { Harmayani et al. (2016) } \\
\text { Heyne (1987) }\end{array}$ \\
\hline "dage benguk" a spontaneous fermentation product & Indonesia, in the past & \\
\hline $\begin{array}{l}\text { "gedebel benguk" (oncom like product, a fermented legume } \\
\text { using Monilia sitophilia) }\end{array}$ & Indonesia, in the past & Heyne (1987) \\
\hline $\begin{array}{l}\text { leafy vegetables from young leaves ( } M \text {. hirsuta W. and A.) } \\
\text { and seeds were consumed as foods eaten with rice or uses as } \\
\text { part of medicines }\end{array}$ & Indonesia, in the past & \\
\hline L-DOPA to relief Parkinson's disease symptoms & na & $\begin{array}{l}\text { Shaw and Bera (1993) } \\
\text { Prakash and Tewari (1999). }\end{array}$ \\
\hline $\begin{array}{l}\text { decoction of Mucuna seeds lowered plasma cholesterol and } \\
\text { lipids in rats }\end{array}$ & na & Iauk et al. (1989) \\
\hline beverage component or consumed as roasted powder & Kenya & Saha and Muli (2000) \\
\hline beverage of coffee mucuna powder & na & Diallo et al. (2002) \\
\hline yogurt & Indonesia & Wanita and Rahayu (2010) \\
\hline beef burger & na & Onweluzo et al. (2004) \\
\hline weaning foods & na & Egounlety (2003) \\
\hline snack & na & Sridhar and Bhat (2007), \\
\hline fried velvet bean snack & Indonesia & Sudiyono (2010) \\
\hline paste or oil soup (stew), & Southern Ghana & \\
\hline roasted snacks (Akpaka Ide) & Southern Ghana & Osei-Bonsuet al (1996) \\
\hline sauce (Akpoko ji/nkashi/Una) & Southern Ghana & \\
\hline gel (Opka), & Southern Ghana & \\
\hline $\begin{array}{l}\text { additives or condiment, e.g. as thickener in sauce or soup, } \\
\text { stabilizer gum or gel, }\end{array}$ & na & Onweluzo et al. (2004) \\
\hline Moi-moi, and fried cake & na & Sridhar and Bhat (2007) \\
\hline porridge & na & $\begin{array}{l}\text { Sridhar and Bhat (2007) } \\
\text { Diallo et al. (2002), }\end{array}$ \\
\hline non soya tempe. & Japan & Higasa et al. (1996) \\
\hline $\begin{array}{l}\text { young leaves and pods were consumed as vegetables; } \\
\text { sometimes also for cattle feeds }\end{array}$ & Indonesia & Heyne (1987) \\
\hline $\begin{array}{l}\text { immature pods and leaves serve as vegetables, while seeds } \\
\text { as condiment and main dish by ethnic groups }\end{array}$ & Nigeria & Adebowale and Lawal (2003b) \\
\hline $\begin{array}{l}\text { daily meal, cooked and ground like mashed velvet beans } \\
\text { M.cochinchinensis and } M \text {. utilis }\end{array}$ & Southern Ghana & \\
\hline $\begin{array}{l}\text { After draining the } \\
\text { cooked water, softened seeds are hulled, ground into paste } \\
\text { and mixed with other ingredients (e.g. chillies, } \\
\text { egg plant, onions, meat or fish) to prepare soup (Asadua and } \\
\text { Nkwan), which is eaten along with starchy } \\
\text { staples. }\end{array}$ & Southern Ghana & Osei-Bonsu et al. (1996) \\
\hline
\end{tabular}


Table 3. Velvet bean diverse nutritional composition based on worldwide researches

\begin{tabular}{|c|c|c|c|c|c|c|c|c|c|c|c|c|}
\hline Nutrients & $\begin{array}{l}\text { Balogun and } \\
\text { Olatidoye } \\
\text { (2012) }\end{array}$ & $\begin{array}{l}\text { Balogun et al. } \\
\text { (2017) }\end{array}$ & $\begin{array}{l}\text { Ravindran and } \\
\text { Ravindran } \\
\text { (1988) }\end{array}$ & $\begin{array}{l}\text { Bhat et al. } \\
\text { (2008) }\end{array}$ & $\begin{array}{l}\text { Sardjono et al. } \\
\text { (2012) }\end{array}$ & $\begin{array}{l}\text { Siddhuraju et al. } \\
\text { (1996) }\end{array}$ & Ezeagu et al. (2003) & $\begin{array}{l}\text { Rajaram and } \\
\text { Janardhanan } \\
\text { (1991) }\end{array}$ & $\begin{array}{l}\text { Ezeagu et al. } \\
\text { (2003) }\end{array}$ & $\begin{array}{l}\text { Mohan and } \\
\text { Janardhanan } \\
\text { (1995) }\end{array}$ & $\begin{array}{l}\text { Afolabi et al. } \\
(1985)\end{array}$ & $\begin{array}{l}\text { Ezeagu et al. } \\
(2003)\end{array}$ \\
\hline origin & Ibadan, Nigeria & $\begin{array}{l}\text { Ibadan, } \\
\text { Nigeria }\end{array}$ & & India & $\begin{array}{l}\text { Yogyakarta, } \\
\text { Indonesia }\end{array}$ & & & & & & & \\
\hline $\begin{array}{l}\text { analyses } \\
\text { locations }\end{array}$ & Nigeria & Indonesia & & India & Indonesia & & & & & & & \\
\hline species & M. utilis black & M. utilis black & M. utilis & M. pruriens & M. pruriens $(\mathrm{L})$ & M. pruriens & M. cochinchinensis & M. gigantea & M. jaspeada & M. monosperma & M. solanei & $\begin{array}{l}\text { M. veracruz } \\
\text { (black) }\end{array}$ \\
\hline \multicolumn{13}{|l|}{ proximate $(\%)$} \\
\hline moisture & $6.02 \pm 0.11$ & $10.33 \pm 0.07$ & - & $9.58 \pm 0.38$ & $\begin{array}{l}9.14^{\text {shell }} \\
10.80^{\text {kernel }}\end{array}$ & - & - & - & - & - & - & - \\
\hline crude protein & $25.65 \pm 0.14$ & $22.67 \pm 0.33$ & 26.40 & $23.04 \pm 0.38$ & & 31.44 & 29.79 & 30.62 & 27.56 & 23.50 & 24.00 & 24.50 \\
\hline crude lipid & $14.52 \pm 0.05^{\text {ether }}$ & $2.12 \pm 0.11^{\text {hexane }}$ & 4.10 & $7.13 \pm 0.17$ & & 6.73 & 6.51 & 9.03 & 4.72 & 14.39 & 6.50 & 6.90 \\
\hline fatty acids ${ }^{0.86 x f a t}$ & 12.49 & & - & - & & - & - & - & - & - & - & - \\
\hline ash & $3.60 \pm 0.01$ & $3.94 \pm 0.02$ & 3.70 & $4.79 \pm 0.72$ & $\begin{array}{l}2.18^{\text {shell }} \\
3.04^{\text {kernel }}\end{array}$ & 4.11 & 4.16 & 5.99 & 3.25 & 3.21 & 3.00 & 3.66 \\
\hline carbohydrate & 42.98 & 60.23 & 59.50 & $57.18 \pm 0.93$ & - & 52.56 & 59.54 & 42.54 & 64.47 & 52.20 & ND & 64.88 \\
\hline crude fiber & $7.23 \pm 0.05$ & - & 6.30 & $7.85 \pm 0.04$ & - & 5.16 & 4.19 & - & 4.43 & 6.79 & 5.30 & 4.27 \\
\hline $\begin{array}{l}\text { in vitro protein } \\
\text { digestibility }\end{array}$ & - & - & - & $50.65 \pm 5.42$ & - & - & - & - & - & - & - & - \\
\hline
\end{tabular}

Table 4. Mineral compositions

\begin{tabular}{|c|c|c|c|c|c|c|c|c|c|}
\hline & $\begin{array}{l}\text { Balogun and } \\
\text { Olatidoye (2012) }\end{array}$ & $\begin{array}{l}\text { Ravindran and } \\
\text { Ravindran (1988) }\end{array}$ & Bhat et al. (2008) & $\begin{array}{l}\text { Mary Josephine } \\
\text { and Janardhanan } \\
\text { (1992) }\end{array}$ & Siddhuraju et al (2000) & Siddhuraju et al (2000) & Ajayi et al (2006) & $\begin{array}{l}\text { Rajaram and } \\
\text { Janardhanan } \\
\text { (1991) }\end{array}$ & Ezeagu et al (2003) \\
\hline $\begin{array}{l}\text { spesies } \\
\text { minerals }(\mathrm{mg} / 100 \mathrm{~g})\end{array}$ & M. utilis (black) & M. utilis & M. pruriens & M. pruriens & M. pruriens var. utilis white & M. pruriens var. utilis black & M. flagellipes & M. gigantea & M. jaspeada \\
\hline$-\mathrm{Ca}$ & $148.88 \pm 0.2$ & 250 & $66.53 \pm 0.92$ & 247 & 87.80 & 104 & 12.80 & 518 & 80 \\
\hline$-\mathrm{Na}$ & $54.46 \pm 0.2$ & 70.00 & $6.15 \pm 2.03$ & 4.10 & 12.70 & 25.70 & 11.10 & 35.30 & - \\
\hline$-\mathrm{K}$ & $1,472.33 \pm 0.2$ & 11,110 & $164 \pm 2.15$ & 2,537 & 1,575 & 1,343 & 1,322 & 2,296 & 8,460 \\
\hline$-\mathrm{Mg}$ & $23.66 \pm 0.3$ & 110 & $42 \pm 0.4$ & 72.40 & 120 & 109 & 58.30 & 506 & 170 \\
\hline$-\mathrm{P}$ & $377.12 \pm 0.2$ & 220 & $245 \pm 11.45$ & 459 & 499 & 376 & - & 194 & 470 \\
\hline$-\mathrm{Fe}$ & $3.44 \pm 0.2$ & 1.30 & $14.63 \pm 1.05$ & 5.19 & 5.79 & 7.47 & 82 & 9.42 & 6,800 \\
\hline$-\mathrm{Zn}$ & $3.46 \pm 0.1$ & 1.00 & $5.7 \pm 1.15$ & 1.71 & 5.26 & 12.20 & 7.30 & 8.24 & 4.60 \\
\hline$-\mathrm{Cu}$ & $0.71 \pm 0.1$ & 0.60 & $2.51 \pm 0.01$ & 0.47 & 2.42 & 1.65 & 2.60 & 1.18 & 1.82 \\
\hline$-\mathrm{Mn}$ & $5.28 \pm 0.1$ & 1.00 & $3.03 \pm 0.12$ & 0.31 & 1.49 & 2.41 & 11.90 & 2.36 & 5.17 \\
\hline$-\mathrm{Se}$ & - & - & $19.43 \pm 4.39$ & - & - & - & - & - & - \\
\hline
\end{tabular}


Table 5. Fatty acid compositions

\begin{tabular}{|c|c|c|c|c|c|c|c|}
\hline & Balogun and Olatidoye (2012) & Bhat et al. (2008) & Siddhuraju et al. (2000)* & Siddhuraju et al. (2000)* & $\begin{array}{l}\text { Siddhuraju et al } \\
(1996)^{*}\end{array}$ & Ajayi et al. (2006)* & Mohan and Janardhanan (1995)* \\
\hline species & M. utilis (black) & M. pruriens & M. pruriens var. utilis white & M. pruriens var. utilis black & M. pruriens & M. flagellipes & M. monosperma \\
\hline \multicolumn{8}{|l|}{ fatty acids $\mathrm{mg} / \mathrm{g}$ lipid } \\
\hline -palmitic & $28.8 \% * *$ & 4.17 & 2.01 & 2.18 & 2.02 & 1.07 & 2.46 \\
\hline -stearic & $18.21^{* *}$ & 0.06 & 0.71 & 0.74 & 0.38 & 0.34 & 1.17 \\
\hline -oleic & $20.12 * *$ & 6.82 & 0.83 & 0.70 & 2.87 & - & 3.08 \\
\hline -linoleic & $26.40 * *$ & - & 4.88 & 4.80 & 3.71 & 1.50 & 2.47 \\
\hline -linolenic & $8.71^{* *}$ & 1.42 & 0.65 & 0.77 & 0.33 & - & 0.47 \\
\hline -behenic & $2.42 * *$ & 0.64 & 0.34 & 0.34 & 0.07 & 0.14 & 0.35 \\
\hline -myristic & - & 0.07 & 0.02 & 0.02 & - & - & - \\
\hline -myristoleic & - & 0.01 & trace & nd & - & - & - \\
\hline -elaidic & - & 2.16 & trace & trace & - & 6.07 & - \\
\hline -linoelaidic & - & 5.82 & - & - & - & - & - \\
\hline -heneicosanoic & - & 0.10 & 0.01 & 0.01 & - & - & - \\
\hline -lauric & - & - & trace & - & - & - & - \\
\hline -arachidic & - & - & 0.14 & 0.45 & 0.18 & - & - \\
\hline -tricosanoic & - & - & - & 0.01 & - & - & - \\
\hline -lignoceric & - & - & 0.09 & 0.09 & - & 0.39 & - \\
\hline -palmitoleic & - & - & 0.03 & 0.03 & 0.17 & - & - \\
\hline -linolelaidic & - & - & 0.27 & 0.15 & - & - & - \\
\hline -eicosadienoic & - & - & 0.01 & 0.01 & - & - & - \\
\hline -eicosenoic & - & - & - & - & - & 0.23 & - \\
\hline -cerotic & - & - & - & 0.01 & - & - & - \\
\hline Oleic:linolenic ratio & $2.31 * *$ & - & - & - & - & - & - \\
\hline Polyunsaturated:saturated & - & 3.20 & 2.02 & 1.83 & 2.67 & 3.54 & 1.51 \\
\hline
\end{tabular}


Table 6. Amino acid compositions

\begin{tabular}{|c|c|c|c|c|c|}
\hline Nutrients & Balogun and Olatidoye (2012) & Bhat et al.(2008) & Adebowale et al (2005) & Siddhuraju et al (1996) & Afolabi et al (1985) \\
\hline $\begin{array}{l}\text { amino acid } \\
\text { composition } \mathrm{g} / 100 \mathrm{~g}\end{array}$ & M. utilis (black) & M. pruriens & M. cochinchinensis & M. pruriens & M. solaneic \\
\hline lysine* & 5.72 & 8.98 & 6.78 & 6.60 & 13.47 \\
\hline histidine* & 3.13 & 3.30 & 2.36 & 3.14 & 1.13 \\
\hline $\operatorname{arginine} *$ & 7.41 & 9.55 & 8.05 & 7.16 & 11.81 \\
\hline aspartic acid & 14.28 & 17.10 & 13.6 & 8.16 & 6.94 \\
\hline glutamic acid & 13.28 & 19.31 & 16.8 & 17.23 & 10.03 \\
\hline glycine & 5.49 & 6.21 & 5.43 & 5.12 & 3.08 \\
\hline valine* & 4.47 & 7.60 & 6.98 & 5.57 & 3.65 \\
\hline methionine* & 0.69 & 0.78 & 1.32 & 1.28 & 1.18 \\
\hline isoleucine* & 7.24 & 8.77 & 9.08 & 4.12 & 2.70 \\
\hline leucine* & 6.14 & 10.42 & 7.27 & 7.85 & 5.16 \\
\hline tyrosine & 3.94 & 7.51 & 5.46 & 4.76 & 11.16 \\
\hline cysteine & 4.52 & 1.61 & 1.04 & 0.84 & 1.72 \\
\hline phenylalanine* & 4.58 & 6.51 & 7.69 & 3.85 & 14.81 \\
\hline serine & 4.53 & 6.08 & 3.45 & 4.10 & 3.32 \\
\hline proline & 3.64 & 7.38 & 13.45 & nd & 3.30 \\
\hline tryptophan & 0.81 & ND & 2.34 & 1.35 & nd \\
\hline alanine & 4.28 & 4.95 & 7.45 & 2.81 & 3.61 \\
\hline threonine* & 3.86 & 5.21 & 5.04 & 3.64 & 2.04 \\
\hline
\end{tabular}

\section{VELVET BEAN PROCESSING AND WATER REQUIREMENTS}

Sterilization of velvet seeds is important (Bhat et al., 2007) that there was fungi growth on the surface of grains including Aspergillus, Fusarium, Eurotium, and yeast. Precaution should be paid for the toxigenic fungi namely Aspergillus flavus, A. niger, and Fusarium sp. by which irradiation at 10 $\mathrm{kGy}$ is sufficiently effective to give better safety levels of fungal contaminations. Hydrothermal treatments, fermentation, and germination are most effective in reducing the antinutrients of velvet seeds (Wanjekeche et al., 2003). Various processing technology has been investigated onto velvet seeds aiming to reduce the toxins.

Accumulated data on velvet bean processing are listed in Table 7 nevertheless literature are scarcely found on an instant ingredient from velvet bean to be modernized based on such huge various processing studied. Indigenous processing often seen as the long successfully tested processing as the prior art for developing instant ingredient oriented for neural diseases already well known obtained from velvet seed products. Besides its prospective and challenging economical values, it is warrants investigation of technological touch to patch a new usage of velvet bean better in environmentally friendly ways.

Aqueous boiling is the best method for L-DOPA removal from velvet seeds (Bressani et al., 2003) and the products developed by International Institute of Tropical Agriculture (IITA), Benin from L-DOPA free velvet beans in Nigeria is accepted by the society (Versteeg et al., 1998). Various processing methods have been tried by investigators to reduce L-DOPA of velvet seeds. Most of the methods employed were based on the use of water, chemicals, and thermal treatments (Bressani, 2002; Diallo and Berhe, 2003; Gilbert, 2002). Dry treatments are the most effective in reducing L-DOPA in velvet seeds and preventing L-DOPA racemization under roasting (Siddhuraju et al., 1996), grilling is considered a better technique than cooking (Dossa et al. 1998). Extreme heating is unfavorable nutritionally due to poor protein availability as well as protein digestibility (Kakade and Evans, 1965) although it removes haemagglutinins. Moisture in seeds plays an important role in the destruction of trypsin inhibitors (Liener and Kakade, 1980). Water imbibes into the kernels through soaking, germination, or hydrothermal processing activates enzymes among others is those which can destroy 
antinutrients in cereal and legume; for instance, phytase which is also favorable when the metabolites of fermentation provide lactic acid (Sandberg, 2002). Meanwhile, fermentation with inoculums helps us with enzymes obtained from microbes to complement the endogenous activated enzymes in the kernels. The best removal of toxins especially those which are water-soluble such as cyanide can be done simply by soaking and removal of testa before boiling, and generally, cooking significantly eliminate $\mathrm{HCN}$ in seeds of Mucuna utilis (Ravindran and Ravindran, 1988).
Based on data of estimated water uses in Table 7 soaking treatment would depend on the waterbeans ratios. The elimination of toxins would necessarily balance with nutritional retentions, in particular, those water-soluble nutrients. With the challenge of limited clean water in the arid areas where the most common locations of velvet bean growth, a high water-beans ratio make the processing unlikely compatible. Heating in reduced water-beans ratios would be more realistic options. Yet, technology such as microwave heating still expensive, and education about its dangers should be introduced for society. Such an idea would only work in the area with the available electrical supply.

Table 7. Processing of velvet beans

\begin{tabular}{|c|c|c|c|c|}
\hline Processing Types & Principal Steps & Results & Minimum water $(\% \mathrm{v} / \mathrm{b} \text { seeds })^{*}$ & References \\
\hline \multicolumn{5}{|c|}{ Germination } \\
\hline \multirow[t]{4}{*}{ Germination } & $\begin{array}{l}\text { i) Sterilized ( } 1 \% \text { mercuri } \\
\text { chloride), rinsed; soaked } \\
\left.\text { (distilled water, } 4{ }^{\circ} \mathrm{C}, 12 \mathrm{~h}\right) \text {; } \\
\text { germinated }(24,48,72,96 \text {, } \\
\left.\text { and } 120 \mathrm{~h} 30{ }^{\circ} \mathrm{C}\right) ; \text { moistened } \\
\text { (distilled water } 12 \mathrm{~h} \text { ), rinsed } \\
\text { the sprout (distilled water) }\end{array}$ & & $\begin{array}{l}\text { Total: } 350 \text { soaking: } 100 \\
\text { moistening: } 25 \text { x } 10 \text { times }=250 \\
\text { rinsing: } \\
100\end{array}$ & $\begin{array}{l}\text { Gurumoorthi and } \\
\text { Uma (2011) }\end{array}$ \\
\hline & ii). Sterilized (ethanol soaking, & & $\begin{array}{l}\text { Total: } \mathbf{1 , 0 0 0} \\
\text { soaking: }\end{array}$ & Mugendi et al. 2010 \\
\hline & $\begin{array}{l}\left.1: 10 \mathrm{w} / \mathrm{v}, 12 \mathrm{~h}, 25^{\circ} \mathrm{C}\right), \\
\text { drained, spread (thick wet } \\
\text { cotton wool), germinated } \\
(\text { dark } 3 \mathrm{~d}) \text {, dehulled, frozen }(- \\
\left.21{ }^{\circ} \mathrm{C} 12 \mathrm{~h}\right) \text { to stop } \\
\text { germination, dried (oven } 50 \\
\left.{ }^{\circ} \mathrm{C}, 24 \mathrm{~h}\right), \text { ground }\left(\leq 500^{\circ} \mathrm{m}\right) \text {, } \\
\text { frozen }\left(-21^{\circ} \mathrm{C}, 12 \mathrm{~h}\right)\end{array}$ & & 1,000 & \\
\hline & $\begin{array}{l}\text { Room temperature, humidified } \\
\text { cotton beds, } 72 \mathrm{~h}\end{array}$ & & Total: 100 & Balogun et al. (2017) \\
\hline $\begin{array}{l}\text { Germination to } \\
\text { reduce trypsin } \\
\text { inhibitor activity }\end{array}$ & Germinated ( 5 and $7 \mathrm{~d}$ ) & $\begin{array}{l}\text { Reduction of trypsin } \\
\text { inhibitors up to } 84.5 \text { and } \\
85.4 \% \text {. }\end{array}$ & insufficient information & $\begin{array}{l}\text { Wanjekeche et al. } \\
\text { (2003) }\end{array}$ \\
\hline $\begin{array}{l}\text { Germination and } \\
\text { boiled }\end{array}$ & $\begin{array}{l}\text { Germinated ( } 5 \text { and } 7 \mathrm{~d}) \text {, } \\
\text { boiled }\end{array}$ & Reduced L-DOPA $38.5 \%$ & $\begin{array}{l}\text { Total } \mathbf{3 0 0 \%} \\
\text { soaking } 100 \% \text {, } \\
\text { boiling: } \\
200 \%\end{array}$ & $\begin{array}{l}\text { Wanjekeche } \text { et al. } \\
(2003)\end{array}$ \\
\hline $\begin{array}{l}\text { Germination and } \\
\text { malting mucuna } \\
\text { seeds to reduce } \\
\text { trypsin inhibitor } \\
\text { activity }\end{array}$ & Germinated ( 2 vs. 6 d) & $\begin{array}{l}\text { Decreased trypsin } \\
\text { inhibitor activity } 1.88 \\
\text { vs. } 0.82 \mathrm{TUI} / \mathrm{mg}) .\end{array}$ & $\begin{array}{l}\text { Total } 300 \% \\
\text { soaking } 100 \%, \\
\text { :daily weting } \\
200 \%\end{array}$ & $\begin{array}{l}\text { Bressani et al. } \\
\text { (2003) }\end{array}$ \\
\hline $\begin{array}{l}\text { Sprouting and oil } \\
\text { frying (Lambadi } \\
\text { Ethnic, India) }\end{array}$ & $\begin{array}{l}\text { Mixed red soil paste }(1: 5 \mathrm{w} / \mathrm{v}) \\
\text { - beans ratios of } 2: 1 \text {; } \\
\text { humidified with wet cloth, } \\
\text { incubated for } 7 \mathrm{~d}, 25^{\circ} \mathrm{C} \text {; } \\
\text { sprout separation, washed, } \\
\text { dried } 85-90^{\circ} \mathrm{C}, 15 \mathrm{~min}\end{array}$ & $\begin{array}{l}\text { Increased free phenolics } \\
\text { by } 4-11 \%(5,81-9,25 \% \\
\text { vs. } 5.24-8.65 \%)\end{array}$ & wetting: $100 \%$; washing: $100 \%$ & $\begin{array}{l}\text { Vadivel and } \\
\text { Biesalski (2012) }\end{array}$ \\
\hline \multicolumn{5}{|c|}{ Fermentation } \\
\hline Fermentation & R.oligosporus fermentation & & $\begin{array}{l}\text { Total } 300 \% \\
\text { soaking } 100 \% \text {, } \\
\text { boiling: } \\
200 \%\end{array}$ & Egounlety (2003) \\
\hline
\end{tabular}




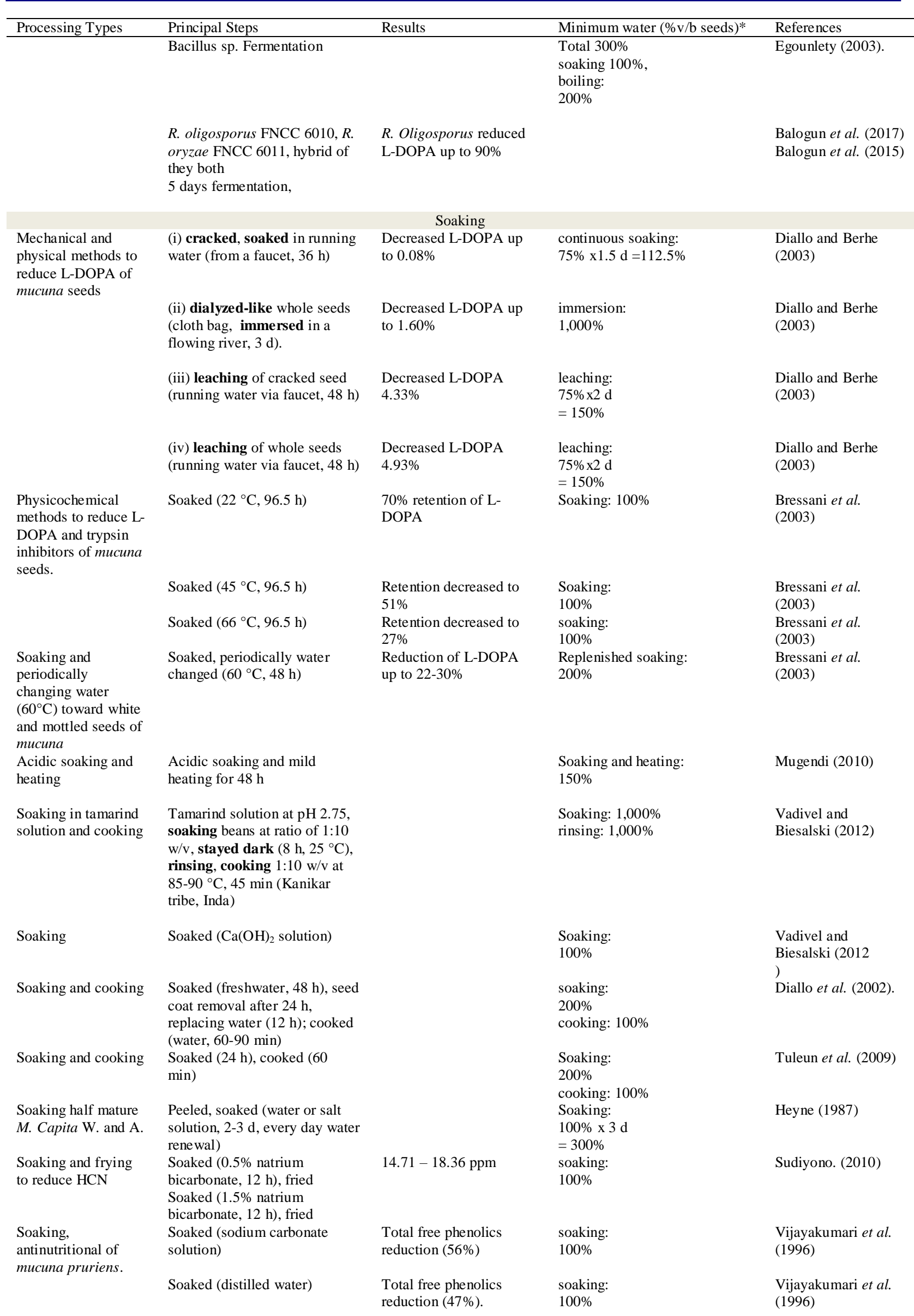




\begin{tabular}{|c|c|c|c|c|}
\hline Processing Types & Principal Steps & Results & Minimum water $(\% \mathrm{v} / \mathrm{b} \text { seeds })^{*}$ & References \\
\hline \multirow[t]{2}{*}{$\begin{array}{l}\text { Soaking to reduce } \\
\text { phytic acid }\end{array}$} & Soaked (distilled water) & Decreased $27 \%$ & $\begin{array}{l}\text { soaking: } \\
100 \%\end{array}$ & Sandberg (2002) \\
\hline & Soaked (sodium bicarbonate) & Decreased $17 \%$ & $\begin{array}{l}\text { soaking: } \\
100 \%\end{array}$ & Sandberg (2002) \\
\hline \multirow[t]{2}{*}{$\begin{array}{l}\text { Soaking and cooking } \\
\text { to reduce phytic acid }\end{array}$} & Soaked, cooked (90 min) & Further reduced $18 \%$ & $\begin{array}{l}\text { soaking and cooking: } \\
150 \%\end{array}$ & Sandberg (2002) \\
\hline & Soaking, autoclaving (45 $\mathrm{min}$ ) & Further reduced $44 \%$ & $\begin{array}{l}\text { soaking and autoclaving: } \\
150 \%\end{array}$ & Sandberg (2002) \\
\hline $\begin{array}{l}\text { Soaking, autoclaving } \\
\text { and cooking mucuna } \\
\text { seeds in to remove } \\
\text { phytic acid }\end{array}$ & $\begin{array}{l}\text { Soaked (various solutions), } \\
\text { autoclaved and cooked }\end{array}$ & $\begin{array}{l}\text { Decline in phytate } \\
\text { content }(27-34 \% \text { and } 38- \\
51 \%)\end{array}$ & $\begin{array}{l}\text { soaking and autoclaving: } \\
150 \%\end{array}$ & $\begin{array}{l}\text { (Siddhuraju and } \\
\text { Becker 2001). }\end{array}$ \\
\hline $\begin{array}{l}\text { Soaking and cooking } \\
\text { to eliminate trypsin } \\
\text { inhibitor }\end{array}$ & $\begin{array}{l}\text { Soaked (water, } 48 \mathrm{~h}) \text {, cooked } \\
(30 \mathrm{~min})\end{array}$ & Complete elimination & $\begin{array}{l}\text { soaking and autoclaving: } \\
150 \%\end{array}$ & $\begin{array}{l}\text { Udedibie and Carlini } \\
\text { (1998) }\end{array}$ \\
\hline
\end{tabular}

\section{Boiling}

Boiling and

dehulling to reduce

L-DOPA $(m$.

Pruriens var. Utilis)

seeds

\author{
Boiling 'Magadi \\ soda' (hydrated \\ sodium carbonate) to \\ reduce L-DOPA in \\ whole mature seeds \\ of mucuna pruriens
}

\section{Boiling}

Boiling

Boiling

Boiling of mucuna cochinchinensis to eliminate

haemagglutinating activities.
Boiled up to $40 \mathrm{~min}$

Boiled (45 min), dehulled

Boiled (45 min), dehulled, soaked $(12 \mathrm{~h})$

Boiled (45 min), dehulled, soaked (12 h), re-soaked (12 h)

Boiled (45 min), dehulled, soaked (12 h), re-soaked (12 h), re-boiled (45 $\mathrm{min}$ )

Boiled in alkaline solution

Reduced L-DOPA by $59.3 \%(5.75 \%$ vs $2.34 \%)$,
Osei-bonsu et al. (1996) Egounlety (2003)
Decrease L-DOPA up to boiling: $6.36 \%$ (raw) to $4.71 \% \quad 100 \%$

$\begin{array}{ll}\text { boiling: } & \text { Osei-bonsu et al. } \\ 100 \% & (1996) \\ \text { boiling: } & \text { Egounlety (2003) } \\ 100 \% & \end{array}$

Reduced L-DOPA up to soaking and boiling: $6.36 \%$ (raw) to $2.29 \% \quad 200 \%$

Reduced L-DOPA up to boiling, resoaking:

$6.36 \%$ (raw) to $1.36 \% \quad 300 \%$

Reduced L-DOPA up to boiling, resoaking: $6.36 \%$ (raw) to $0.64 \% \quad 400 \%$

Egounlety (2003)

Egounlety (2003)

Egounlety (2003)

boiling: $100 \%$

Wanjekeche et al. (2003)
Boiled in cob ash solution

Boiled in citric acid solution

Boiled in bean stover ash solution

Boiled seeds in water

Boiled $\left(90 \mathrm{~min}, 100-105^{\circ} \mathrm{C}\right)$
Reduced by $58.1 \%(5.75$ vs. $2.81 \%$ ).

Reduced by $49.7 \%$ (5.75 boiling: $100 \%$ vs. $2.89 \%$ ).

Reduced by $47.4 \%$ (5.75 boiling: $100 \%$ vs. $3.02 \%$ ).

Reduced L-DOPA up to boiling: $100 \%$ $24.9 \%$

Failed to eliminate all boiling: $100 \%$

boiling: $100 \%$
Wanjekeche et al. (2003)

Wanjekeche et al. (2003)

Wanjekeche et al. (2003)

Wanjekeche et al. (2003)

Ukachukwu and Obioha (2000) 


\begin{tabular}{|c|c|c|c|c|}
\hline Processing Types & Principal Steps & Results & Minimum water (\%v/b seeds)* & References \\
\hline Boiling velvet bean & $\begin{array}{l}\text { Soaked (plain water, } 24 \mathrm{~h} \text {, } \\
\text { room temperature), washed, } \\
\text { put into boiling water, boiled } \\
20,40 \text {, and } 60 \text { min),drained, } \\
\text { sundried }(4 \mathrm{~d}) \text {, ground }\end{array}$ & $\begin{array}{l}\text { Selenium content }(0.24 \\
\text { ppm vs. } 0.09-0.12 \mathrm{ppm}) \text {, } \\
\text { iron }(103 \mathrm{ppm} \text { vs. } 70-90 \\
\text { ppm), and phosphorous } \\
\text { ( } 0.41 \% \text { vs. } 0.26-0.34 \%) \\
\text { among boiling time } \\
\text { course there were } \\
\text { relatively insignificant. } \\
\text { Crude protein }(29.37 \% \\
\text { dm vs. } 27.93-28.27 \%) \text {, } \\
\text { lipid }(5.9 \% \text { vs. } 3.17- \\
4.53 \%) \text {, and ash }(4.43 \% \\
\text { vs. } 6.68-8.78 \%) \text {. } \\
\text { Reduced almost half } \\
\text { essential amino acids due } \\
\text { to the length of boiling } \\
\text { time. } \\
\text { HCN } 33.46 \mathrm{mg} / \mathrm{kg} \mathrm{DM} \\
\text { vs. } 29.02-32.04 \mathrm{mg} / \mathrm{kg}) \text {, } \\
\text { tannin }(1.41 \mathrm{~g} / \mathrm{kg} \\
\text { vs.0.99-1.16 } / \mathrm{kg}), \\
\text { trypsin inhibitor activity } \\
(33.59 \mathrm{TUI} / \mathrm{mg} \mathrm{vs.} 17.77- \\
23.03 \mathrm{TUI} / \mathrm{mg}), \text { and } \\
\text { oxalates }(1.95 \mathrm{~g} / \mathrm{kg} \mathrm{dm} \\
\text { vs. } 1.38-1.90 \mathrm{~g} / \mathrm{kg}) \text {. }\end{array}$ & $\begin{array}{l}\text { soaking, washing, boiling: } \\
300 \%\end{array}$ & Tuleun et al. (2009) \\
\hline
\end{tabular}

Pound and ground

Microwave assisted heating

Dehulling/soaking and irradiating mucuna seeds Gamma irradiation

Processing methods suitable for household and community level preparations to reduce L-DOPA of muсuna (raw white $3.75 \%$ ), (raw speckled $3.90 \%$ ), (raw black $4.36 \%$ ) (pre-soaked speckled $4.02 \%)$

Various cooking treatments to reduce L-DOPA in mucuna seeds.
Pounded, cracked, drained, hulled, ground

Microwave heated $\left(130{ }^{\circ} \mathrm{C}\right)$ overnight soaked, microwave heated $\left(130{ }^{\circ} \mathrm{C}\right)$

Dehulled, irradiated

Soaked, irradiated

Boiled (water)
Reduced L-DOPA up to
$48.5 \%$ ( 4.02 vs. $2.07 \%$ )

insufficient information

Increased L-DOPA

Reduced total phenolics (up to $80 \%$ )

boiling: $100 \%$

Osei-bonsu et al. (1996)

Kala and Mohan (2012)

Siddhuraju et al (2000)

Bhat et al. (2007)

Nyirenda et al. (2003) $57 \%$ (4.02 to $1.72 \%$ )

Microwave, vapor, in various water solutions at ph 3, 6, 7, and 11 , cooking in alkaline sodium hydroxide/potassium hydroxide/calcium hydroxide Cooked (calcium hydroxide, $\mathrm{pH} 9$ ), washed in hot water
Reduced L-DOPA up to Extracted L-DOPA

approximately $90 \%$ (4.02\% vs. $0.39 \%)$. Reduced L-DOPA up to $67 \%$.

Reduced L-DOPA up to $54 \%$ (4.02 vs. $1.86 \%$ )

Not effective

boiling: $100 \%$

boiling and resoaking:

$3,000 \%$

boiling and resoaking: $3,000 \%$

soaking: $3,000 \%$

soaking an cooking: $200 \%$

Reduction up to $80.4 \%$ total $=300 \%$

cooking:

$100 \%$

washing: $200 \%$
Nyirenda et al. (2003)

Nyirenda et al. (2003)

Nyirenda et al. (2003)

Nyirenda et al. (2003)

Garcia-Echeverria and Bressani (2006)

Garcia-Echeverria and Bressani (2006) 


\begin{tabular}{|c|c|c|c|c|}
\hline Processing Types & Principal Steps & Results & Minimum water (\%v/b seeds)* & References \\
\hline $\begin{array}{l}\text { Cooked yellow } \\
\text { powder as soup } \\
\text { thickener }\end{array}$ & $\begin{array}{l}\text { Cracked (hitting with a hard } \\
\text { object), cooked, hulled, } \\
\text { ground, mixed with red palm } \\
\text { oil }\end{array}$ & & $\begin{array}{l}\text { cooking: } \\
100 \%\end{array}$ & Ezueh (1997) \\
\hline $\begin{array}{l}\text { Cooking and } \\
\text { autoclaving to reduce } \\
\text { hemagglutinating } \\
\text { activity }\end{array}$ & & $\begin{array}{l}\text { Reduced } \\
\text { hemagglutinating activity } \\
\text { up to } 89-99 \%\end{array}$ & $\begin{array}{l}\text { cooking and autoclaving: } \\
150 \%\end{array}$ & $\begin{array}{l}\text { Vijayakumari et al. } \\
\text { (1996). }\end{array}$ \\
\hline $\begin{array}{l}\text { Cooking to eliminate } \\
\text { haemagglutinin } \\
\text { activity oin } M . \\
\text { cochinchinensis } \\
\text { seeds }\end{array}$ & Cooked $\left(3 \mathrm{~h}, 100{ }^{\circ} \mathrm{C}\right)$ & $\begin{array}{l}\text { Eliminated } \\
\text { haemagglutinin activity }\end{array}$ & $\begin{array}{l}\text { cooking: } \\
100 \%\end{array}$ & Onwuka (1997). \\
\hline $\begin{array}{l}\text { Cooking to inactivate } \\
\text { trypsin inhibitors in } \\
\text { mисипа seeds }\end{array}$ & Cooked $\left(1 \mathrm{~h}, 96^{\circ} \mathrm{C}\right)$ & Completely eliminated & $\begin{array}{l}\text { cooking: } \\
100 \%\end{array}$ & $\begin{array}{l}\text { Udedibie and Carlini } \\
\text { (1998) }\end{array}$ \\
\hline $\begin{array}{l}\text { Cooking to decrease } \\
\text { antitryptic activity }\end{array}$ & & Totally eliminated & $\begin{array}{l}\text { cooking: } \\
100 \%\end{array}$ & $\begin{array}{l}\text { Ravindran and } \\
\text { Ravindran (1988). }\end{array}$ \\
\hline $\begin{array}{l}\text { Cooking to reduce } \\
\text { cyanide, in mucuna }\end{array}$ & Cooked & $\begin{array}{l}\text { Reduces cyanide up to } \\
46 \%\end{array}$ & $\begin{array}{l}\text { cooking: } \\
100 \%\end{array}$ & Montgomery (1980) \\
\hline Toasted flour & Toasted (5-10 min), ground & & $\begin{array}{l}\text { sanitary need: } \\
25 \%\end{array}$ & $\begin{array}{l}\text { Sridhar and Bhat } \\
\text { (2007) }\end{array}$ \\
\hline $\begin{array}{l}\text { Toasting to eliminate } \\
\text { trypsin inhibitors of } \\
\text { M. Utilis (raw, } 2,170 \\
\text { TIU/g) }\end{array}$ & & $\begin{array}{l}\text { Elimination of } 42 \% \\
\text { (6,979 vs. } 11,865 \mathrm{TIU} / \mathrm{g}) \text {. }\end{array}$ & $\begin{array}{l}\text { sanitary need: } \\
25 \%\end{array}$ & $\begin{array}{l}\text { Ravindran and } \\
\text { Ravindran (1988) }\end{array}$ \\
\hline Roasting & Roasted $\left(100{ }^{\circ} \mathrm{C}\right)$ & Increased L-DOPA & $\begin{array}{l}\text { sanitary need: } \\
25 \%\end{array}$ & $\begin{array}{l}\text { Mugendi and Njagi } \\
\text { (2010) }\end{array}$ \\
\hline $\begin{array}{l}\text { Roasting of mисипа } \\
\text { seeds to reduce } \\
\text { trypsin inhibitor } \\
\text { activity }\end{array}$ & Roasted (30 min) & $\begin{array}{l}\text { Reduced the trypsin } \\
\text { inhibitors (raw } 18.90 \text { vs. } \\
1.58 \mathrm{TUI} / \mathrm{mg} \text { ). }\end{array}$ & $\begin{array}{l}\text { sanitary need: } \\
25 \%\end{array}$ & $\begin{array}{l}\text { Bressani et al. } \\
(2003)\end{array}$ \\
\hline $\begin{array}{l}\text { Roasting and } \\
\text { dehulling to reduce } \\
\text { cyanide, (raw } 18.6 \\
\mathrm{mg} / \mathrm{kg} \text { ) }\end{array}$ & $\begin{array}{l}\text { Roasted } \\
\text { Dehulled, roasted }\end{array}$ & $\begin{array}{l}\text { Complete removal of } \\
\text { HCN }\end{array}$ & $\begin{array}{l}\text { sanitary need: } \\
25 \%\end{array}$ & $\begin{array}{l}\text { Agbede and Aletor } \\
(2005)\end{array}$ \\
\hline $\begin{array}{l}\text { Dry heat treatment } \\
\text { and autoclaving } M \text {. } \\
\text { pruriens to reduce } \\
\text { phytic acid }\end{array}$ & Dry heated, autoclaved & $\begin{array}{l}\text { Reduced the phytic acid } \\
(36 \% \text { and } 47 \%)\end{array}$ & $\begin{array}{l}\text { total: } \mathbf{7 5 \%} \\
\text { sanitary need: } \\
25 \% \\
\text { autoclaving: } \\
50 \%\end{array}$ & $\begin{array}{l}\text { Siddhuraju et al. } \\
\text { (1996) }\end{array}$ \\
\hline $\begin{array}{l}\text { Dry heat treatment to } \\
\text { reduce hydrogen } \\
\text { cyanide (HCN) } M \text {. } \\
\text { Pruriens seeds }\end{array}$ & Dry heated & Reduced HCN (67\%) & $\begin{array}{l}\text { sanitary need: } \\
25 \%\end{array}$ & $\begin{array}{l}\text { Siddhuraju et al. } \\
\text { (1996) }\end{array}$ \\
\hline $\begin{array}{l}\text { Autoclaving to } \\
\text { reduce hydrogen } \\
\text { cyanide (HCN) } M \text {. } \\
\text { pruriens seeds }\end{array}$ & Autoclaved & Reduced HCN (68\%) & $\begin{array}{l}\text { autoclaving: } \\
100 \%\end{array}$ & $\begin{array}{l}\text { Siddhuraju et al. } \\
\text { (1996) }\end{array}$ \\
\hline $\begin{array}{l}\text { Autoclaving to } \\
\text { reduce cyanide }\end{array}$ & Autoclaved & $\begin{array}{l}\text { Reduces cyanide up to } \\
75 \% \text {. }\end{array}$ & $\begin{array}{l}\text { autoclaving: } \\
100 \%\end{array}$ & Montgomery (1980). \\
\hline $\begin{array}{l}\text { Autoclaving } \\
\text { antinutritional of } M . \\
\text { pruriens to reduce } \\
\text { tannins }\end{array}$ & Autoclaved (45 min) & Reduced tannins $(71 \%)$ & $\begin{array}{l}\text { autoclaving: } \\
100 \%\end{array}$ & $\begin{array}{l}\text { Vijayakumari et al. } \\
\text { (1996) }\end{array}$ \\
\hline $\begin{array}{l}\text { Autoclaving, } \\
\text { dehulling and } \\
\text { roasting, dehulling } \\
\text { and soaking }\end{array}$ & $\begin{array}{l}\text { Autoclaved (raw/ dehulled), } \\
\text { Dehulled, roasted } \\
\text { Dehulled, soaked (urea) }\end{array}$ & $\begin{array}{l}\text { Completely removed } \\
\text { trypsin inhibition activity } \\
\text { (raw } 25.3 \mathrm{mg} / \mathrm{g} \text { ) }\end{array}$ & $\begin{array}{l}\text { autoclaving: } \\
100 \% \\
\text { autoclaving and soaking: } \\
150 \%\end{array}$ & $\begin{array}{l}\text { Agbede and Aletor } \\
(2005)\end{array}$ \\
\hline $\begin{array}{l}\text { Dehulling and } \\
\text { cooking/roasting to } \\
\text { reduced lectin } M \text {. } \\
\text { pruriens seed flours. }\end{array}$ & $\begin{array}{l}\text { Dehulled, cooked } \\
\text { Dehulled, roasted }\end{array}$ & $\begin{array}{l}\text { Completely eliminated } \\
\text { lectin (raw } 4.0 \mathrm{HU} / \mathrm{mg} \text { ). }\end{array}$ & $\begin{array}{l}\text { cooking: } \\
100 \%\end{array}$ & $\begin{array}{l}\text { Agbede and Aletor } \\
(2005)\end{array}$ \\
\hline $\begin{array}{l}\text { Dehulling and } \\
\text { roasting to reduced } \\
\text { phytin and phytin } \\
\text { phosphorus mucuna } \\
\text { seeds (raw } 15.3 \text { and } \\
4.3 \mathrm{mg} / 100 \mathrm{~g} \text { ) }\end{array}$ & Dehulled, roasted seed flours & $\begin{array}{l}\text { Reduced phytin and } \\
\text { phytin phosphorus ( } 6.0 \\
\text { mg/100 g). }\end{array}$ & $\begin{array}{l}\text { sanitary need: } \\
25 \%\end{array}$ & $\begin{array}{l}\text { Agbede and Aletor } \\
(2005)\end{array}$ \\
\hline
\end{tabular}




\begin{tabular}{|c|c|c|c|c|}
\hline Processing Types & Principal Steps & Results & Minimum water $(\% \mathrm{v} / \mathrm{b} \text { seeds })^{*}$ & References \\
\hline $\begin{array}{l}\text { "Gedebel benguk" } \\
\text { making }\end{array}$ & $\begin{array}{l}\text { Boiled completely cooked, } \\
\text { removed peel, placed into a } \\
\text { bamboo basket for 'dialyzing } \\
\text { like' process using the flowing } \\
\text { clean water, chopped finely, } \\
\text { steamed, mashed, put into a } \\
\text { mould and placed onto banana } \\
\text { leaves, covered with particular } \\
\text { bamboo leaves for spontaneous } \\
\text { fermentation (uncovered area } \\
\text { mixed with Rhizopus sp. } \\
\text { fermentation). }\end{array}$ & $\begin{array}{l}\text { no data on chemical } \\
\text { effects }\end{array}$ & $\begin{array}{l}\text { total: } \mathbf{6 5 0} \% \\
\text { boiling: } \\
100 \% \\
\text { dializing-like: } 500 \% \\
\text { steaming: } 50 \%\end{array}$ & Heyne (1987) \\
\hline $\begin{array}{l}\text { "Dage benguk" } \\
\text { making }\end{array}$ & $\begin{array}{l}\text { Steamed completely cooked, } \\
\text { removed peel, soaked (water, } \\
48 \text { h), placed into a small } \\
\text { basket (covered with banana } \\
\text { leaves, } 3-4 \text { d), re-steamed }\end{array}$ & $\begin{array}{l}\text { no data on chemical } \\
\text { effects }\end{array}$ & $\begin{array}{l}\text { total= } \mathbf{3 0 0 \%} \\
\text { steaming: } \\
50 \% \\
\text { cooking: } 100 \% \\
\text { soaking: } 100 \% \\
\text { resteaming: } \\
50 \%\end{array}$ & Heyne (1987) \\
\hline $\begin{array}{l}\text { Steaming and } \\
\text { foaming }\end{array}$ & Steamed (lipidic foam) & Safe (tasty) & $\begin{array}{l}\text { steaming: } \\
50 \%\end{array}$ & Heyne (1987) \\
\hline
\end{tabular}

In Indonesia, mucuna seeds were consumed depends on the variety. M. hirsuta W. and A. indicate the needs of a lot of water to make the seed edible: (a) the young seed is boiled, peeled, and washed thoroughly with fresh water and then steamed; (b) mature seed is broken to separate the seed from the peel and the kernel can be eaten directly (M. hirsuta $\mathrm{W}$. and A.) or the mature seed is boiled, its peel is removed, soaked into water for $24 \mathrm{~h}$ with water is renewed every 8-12 $\mathrm{h}$. Any processing should involve water to soak up to $48 \mathrm{~h}$. Generally, old documents have recorded that soaking is the key processing to avoid a headache from improper removal of velvet bean components during processing (Heyne, 1987).

Antioxidant capacity measured by DPPH methods for the velvet bean flour obtained from germination is superior compared to Rhizopus spp FNCC 610 and 6011 fermentation, as well their hybrid (Balogun et al., 2017). Meanwhile, Rhizopus oligosporus has acted well-reducing L-DOPA in the velvet bean after 5 days fermentation (Balogun et al., 2015) which reached $90 \%$. In this case, the seed coat of black velvet bean does not only contribute more scavengers (Balogun et al., 2017) but also becomes important provision to supply mineral intakes (iron $7.9 \mathrm{mg} / 100 \mathrm{~g}$ dry weights, and other minerals comparable to those of soybean) (Mugendi et al., 2010a) and dietary fibers for human as well. L-DOPA is concluded existing in the seed coat higher $(6.98 \%)$ in the unhulled raw velvet beans than dehulled one $(5.71 \%)$ (Mugendi et al., 2010b). Therefore, it is recommended to involve the seed coat of the velvet bean in commercial production of health ingredients from the bean.

Then, germination is outnumbered other methods for other choices. It resulted in mild eradication of antinutrients, phenolics, and L-DOPA (6.77$43.78 \%$ ). Indeed, there are several processes capable of eliminating L-DOPA or antinutrients at the safe levels recommended by WHO/FAO ( $\leq 0.1 \%)$ (Mugendi et al., 2010b). Concerning priority of the Parkinson disease prevention and long term preventive-curative uses, the health ingredients expected to be produced is flour containing sufficient levels of L-DOPA i.e. 4-6\% or $30 \mathrm{~g}$ mucuna seed powder.(Katzenschlager et al., 2004) Because L-DOPA is thermally resistant (Mugendi et al., 2010b), to decreasing other antinutrient factors (i.e. the well-known L-DOPA, total free phenolics, tannins, haemagglutinin, trypsin and chymotrypsin inhibitors, antivitamins, protease inhibitors, phytic acid, flatulence factors, saponins, and hydrogen cyanide) more after germinating, it is easier to treating further by any types of food processing. Nevertheless, it is not recommended to have a monotonous diet based on velvet bean food products because histological examination indicates inflamed liver and kidney even though the L-DOPA has reached safety levels (Mugendi et al., 2010a). A study using velvet bean as part of chicken feeds (Tuleun et al., 2009) show changes due to boiling resulted in broiler 
biochemistry effects of higher weight gain (26.32 $\mathrm{g} / \mathrm{d}$ vs. 34.68-39.79 g/d), protein intake $(16.79 \mathrm{~g} / \mathrm{d}$ vs. 17.50-18.93 g/d) and PER (1.55 vs. 1.99-2.10). Soaking the beans for $24 \mathrm{~h}$ and cooking for $60 \mathrm{~min}$ is recommended as adequate to improve the nutritional quality of velvet beans. In an attempt to reduce water uses, germination and fermentation are the best to simultaneously change several antinutritional factors. To make it standardized processing then the use of the controlled instrument is important therefore the temperature and humidity are at the right levels to make sure biochemical changes consistently.

\section{LOWER WATERED PROCESSING ALTERNATIVES}

Malting technology for preparing malt in beer making is the most established technology of seedling. Germination was adopted from malting technology (Dabija 2012): velvet beans were soaked in 1:2 ratios of beans: water for 4-6 $\mathrm{h}$ until moisture content of $30 \%$; sprayed wet for $16 \mathrm{~h}$ until moisture content (using infrared moisture tester) MC 31-32\%; soaked for 2-4 h until 38\% MC; sprayed wet for $14-18 \mathrm{~h}$ to reach $40 \%$. Then the beans entered germination step at humidity levels of $75 \%$ and $85 \%$, temperature $15{ }^{\circ} \mathrm{C}$ and $20{ }^{\circ} \mathrm{C}$ steeping until it started to be rootlets. At this stage, cold water was introduced to reduce the temperature and MC content increased to 50-52\%. Total water estimated in malting technology is presented in Figure 2 (written in bold letters) and the total water requirement is $370 \%$. In wheat milling technology, to moisten grains before commencing grinding/milling is important and at this stage variations of kernel characteristics become crucial.

Another alternative is steaming of velvet bean: to soak velvet bean at MC of $40 \%$ was steamed at 80 ${ }^{\circ} \mathrm{C}$ for $20 \mathrm{~min}, 30 \mathrm{~min}$, and $45 \mathrm{~min}$ which was placed making bed beans against the steam flow. Steaming has limited water supply for gelatinization therefore L-DOPA leaching will be controlled. The effect of processing and water needs is listed in Table 8.
It has not been available research on instant food ingredients aiming to service Parkinson's disease sufferers. Meanwhile, relationship between LDOPA and germination time course is expressed as $\mathrm{Y}=0.175+2.057 \mathrm{X}-1.548 \mathrm{X}^{2}\left(\mathrm{R}^{2}=0.791\right)$ (Gurumoorthi et al., 2011). From Table 8 the priority is that L-DOPA removal is controlled to the optimal levels for dietary L-DOPA at safe levels. The second priority is antitrypsin and cyanides compulsorily reach maximum reduction. Thus alternatives mucuna processing is combinations of aquadest soaking, germination, boiling, cooking especially those involving oils and foaming (Heyne, 1987), roasting, oil frying (Sudiyono, 2010). To achieve the goal of instant ingredients thus foaming and steaming must be involved during processing. Possible products developed are suggested in the present paper include mayonnaise, ice cream, marshmallow, meringue filler, oil soups, or foamed dried flour.

$1^{\text {st }}$ soaking $(1: 2 \mathrm{w} / \mathrm{v}): \mathbf{2 0 0} \%$

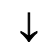

$1^{\text {st }}$ spraying to increase $2 \%$ water content would need $\mathbf{1 0 \%}$

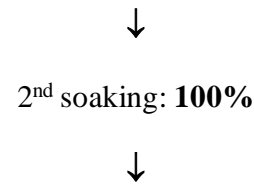

$2^{\text {nd }}$ spraying to increase $2 \%$ water content would need $\mathbf{1 0 \%}$

\section{$\downarrow$}

final rinsing to increase $10 \%$ water content would need $\mathbf{5 0 \%}$

Figure 2. Estimation of malting technology of wheat 
Table 8. Processing effects on mucuna seeds components and water needs during processing

\begin{tabular}{|c|c|c|c|c|}
\hline No. & Processing & Components & Reduction (\%) & Water (\%) \\
\hline 1. & Soaking-germination & Antitrypsin & 85.4 & 350 \\
\hline 2. & Germination-malting & Antitrypsin & 43.62 & 370 \\
\hline 3. & Water boiling & Antitrypsin & 89.7 & 100 \\
\hline 4. & Soaking-cooking & Antitrypsin & 100 & 150 \\
\hline 5. & Cooking & Antitrypsin & 100 & 100 \\
\hline 6. & Cooking & Antitrypsin & 100 & 100 \\
\hline 7. & Toasting & Antitrypsin & 42 & 0 \\
\hline 8. & Roasting & Antitrypsin & 8.36 & 0 \\
\hline 9. & Autoclaving (raw/dehulled) & Antitrypsin & 100 & 50 \\
\hline 10. & Dehulling-roasting & Antitrypsin & 100 & 0 \\
\hline 11. & Dehulling-urea soaking & Antitrypsin & 100 & 100 \\
\hline 10. & Fermentation & L-DOPA & 90 & 100 \\
\hline 12. & Cracking-continuous soaking (in the river) & L-DOPA & 0.08 & unlimited \\
\hline 13. & Dialyzed-like & L-DOPA & 1.6 & 500 \\
\hline 14. & Cracking-continuous leaching & L-DOPA & 4.33 & 112.5 \\
\hline 15. & Leaching & L-DOPA & 4.93 & 1,000 \\
\hline 16. & Soaking $22{ }^{\circ} \mathrm{C}$ & L-DOPA & 30 & 150 \\
\hline 17. & Soaking $45^{\circ} \mathrm{C}$ & L-DOPA & 49 & 100 \\
\hline 18. & Soaking $66{ }^{\circ} \mathrm{C}$ & L-DOPA & 73 & 100 \\
\hline 19. & Soaking, changing water $60^{\circ} \mathrm{C}$ & L-DOPA & 30 & 200 \\
\hline 20. & Soaking-germination-boiling & L-DOPA & 38.5 & 1,000 \\
\hline 21. & Boiling-dehulling & L-DOPA & 24 & 100 \\
\hline 22. & Boiling-dehulling-soaking & L-DOPA & 64 & 200 \\
\hline 23. & Boiling-dehulling-resoaking & L-DOPA & 79 & 300 \\
\hline 24. & Boiling-dehulling-resoaking-reboiling & L-DOPA & 90 & 400 \\
\hline 25. & Alkaline boiling & L-DOPA & 59.3 & 100 \\
\hline 26. & Cob ash boiling & L-DOPA & 58.1 & 100 \\
\hline 27. & Citric acid boiling & L-DOPA & 49.7 & 100 \\
\hline 28. & Bean stover ash boiling & L-DOPA & 47.4 & 100 \\
\hline 29. & Water boiling & L-DOPA & 24.9 & 100 \\
\hline 30. & Water boiling & L-DOPA & 48.5 & 100 \\
\hline 31. & Water boiling-grits & L-DOPA & 57 & 100 \\
\hline 32. & $\mathrm{Na}$ (bicarbonate) soaking-grits-boiling-resoaking & L-DOPA & 90 & 3,000 \\
\hline 33. & $\mathrm{Na}$ (bicarbonate) soaking-boiling-resoaking & L-DOPA & 67 & 3,000 \\
\hline 34. & Water soaking-grits & L-DOPA & 54 & 3,000 \\
\hline 35. & $\mathrm{Ca}$ (hydroxide, ph 9) cooking, hot water washing & L-DOPA & 80.4 & 300 \\
\hline 36. & Soaking-Na(carbonate) & Total phenol & 56 & 150 \\
\hline 37. & Soaking-aquadest & Total phenol & 47 & 100 \\
\hline 38. & Dehulling/soaking-irradiating & Total phenol & 80 & 100 \\
\hline 39. & Soaking-aquadest & Phytic acid & 27 & 200 \\
\hline 40. & Soaking-Na(carbonate) & Phytic acid & 17 & 150 \\
\hline 41. & Soaking-cooking & Phytic acid & 18 & 150 \\
\hline 42. & Soaking-autoclaving & Phytic acid & 44 & 150 \\
\hline
\end{tabular}




\begin{tabular}{|c|c|c|c|c|}
\hline No. & Processing & Components & Reduction $(\%)$ & Water $(\%)$ \\
\hline 43. & Soaking-autoclaving-cooking & Phytic acid & 51 & 150 \\
\hline 44. & Dry heating & Phytic acid & 36 & 0 \\
\hline 45. & Autoclaving & Phytic acid & 47 & 50 \\
\hline 46. & Dehulling-roasting & Phytic substances & 70.4 & 0 \\
\hline 47. & Autoclaving & Hemagglutinating activity & 99 & 50 \\
\hline 48. & Cooking & Hemagglutinating activity & 89 & 100 \\
\hline 49. & Cooking & Hemagglutinating activity & 100 & 100 \\
\hline 50 & Cooking & Cynide & 46 & 100 \\
\hline 51. & Roasting & Cynide & 100 & 0 \\
\hline 52. & Roasting-dehulling & Cynide & 100 & 0 \\
\hline 53. & Dry heating & Cynide & 67 & 0 \\
\hline 54. & Autoclaving & Cynide & 68 & 50 \\
\hline 55. & Autoclaving & Cynide & 75 & 50 \\
\hline 56. & Dehulling-cooking & Leptin & 100 & 100 \\
\hline 57. & Dehulling-roasting & Leptin & 100 & 0 \\
\hline 58. & Soaking-washing-boiling-draining-drying & Micronutrients & 50 & 300 \\
\hline 59. & Autoclaving & Tannin & 71 & 50 \\
\hline
\end{tabular}

\section{PHYSICOCHEMICAL PROPERTIES OF VELVET BEAN FLOUR FROM VARIOUS PROCESSING}

Data in Table 9 indicate the effects of the cooking of various techniques. Dehulling increased water absorption capacity as well as oil absorption capacity, foam stability, and viscosity but it reduced gel, emulsion, and foaming capacity, swelling power, bulk density, and $\mathrm{pH}$ (Balogun and Olatidoye, 2012). Defatting treatment on various mucuna members indicated increasing water absorption capacity and foam capacity but reducing emulsion capacity (Adebowale et al., 2005). Heating increased water and oil absorption capacity but reduced emulsion and foaming capacity whereas foam stability are relatively similar (Ahenkora et al., 1999). Acetylating of velvet bean flour showed an increase in water and oil absorption capacity while oxidizing only increased solubility of the flour quite profoundly (Adebowale and Lawal, 2003).

Irradiation can be considered to preserve almost all physicochemical properties with better cooking time. Irradiation reduces the texture of raw velvet beans at dosages >7.5 kGy might be due to polymer degradations, solubility, turgor, and moisture losses (Bhat et al., 2007). Irradiation gives better sterilization on microbial contamination on the seed surfaces (Bhat et al., 2007) using ionized irradiation and electron beam irradiation. These technologies are expensive and several important properties similar to those of whole velvet bean in the study of Balogun and Olatidoye (2012). Shorter cooking time after irradiation treatments may reduce energy for cooking but it is unclear if the water requirements for cooking would also be reduced. The availability of lipid/oil in velvet bean food preparation that is considered safe (Vadivel and Bielsaski, 2012) gives clues that defatting is unnecessarily carried out. Lipid is required to 'tame' itching component and to make better palatability (Heyne, 1987). Chemical modification through acetylating and oxidizing may be useful to improve the solubility of velvet bean products during processing. However, washing steps after treating the beans with the chemicals would consume a lot of water. The left problem using the whole bean is more likely to get a long time cooking due to the whole beans to have a high bulk density (Seena and Sridhar, 2005). Therefore, it is noteworthy to keep 
Table 9. Physicochemical properties

\begin{tabular}{|c|c|c|c|c|c|c|c|c|c|c|c|}
\hline $\begin{array}{c}\text { Physicochem. } \\
\text { properties }\end{array}$ & \multicolumn{2}{|c|}{ Balogun and Olatidoye (2012) } & \multicolumn{2}{|c|}{ Adebowale et al. (2005) } & \multicolumn{2}{|c|}{ Ahenkora et al. (1999) } & \multicolumn{3}{|c|}{ Adebowale and Lawal (2003) } & \multicolumn{2}{|c|}{ Bhat et al. (2008) } \\
\hline \multirow[t]{2}{*}{ species } & \multicolumn{2}{|c|}{ Mucuna utilis, } & $\begin{array}{r}\text { M.coch } \\
\text { deerigeanc } \\
\text { rajada, } M . \\
\text { and } M . v\end{array}$ & $\begin{array}{l}\text { ensis, } M . \\
\text { rruriens, } M \text {. } \\
\text { ruz (mottle) } \\
u z \text { (white) }\end{array}$ & & & & & & \multicolumn{2}{|c|}{ Mucuna pruriens } \\
\hline & whole & dehulled & full fat & defatted & raw & heated & native & acetylation & oxidized & raw & irradiated (2.5-30 kGy) \\
\hline WAC & 2.05 & 2.45 & $1.40-2.20$ & higher & $140 \%$ & $156 \%$ & 1.71 & 121 & & ca. $2.2 \mathrm{~mL} / \mathrm{g}$ & slightly increased \\
\hline $\mathrm{OAC}$ & 1.19 & 1.45 & & & $76 \%$ & $86 \%$ & & increased & reduced & ca. $1.5 \mathrm{~mL} \mathrm{~g}$ & slightly increased \\
\hline GC (\%) & 4.12 & 3.85 & $14-20$ & & & & & & & & \\
\hline EC & 24.42 & 22.40 & $78-90 \%$ & $56-68 \%$ & $60 \%$ & $50 \%$ & & & & ca. $50 \%$ & relatively similar \\
\hline ES & & & & & & & & & & ca. $90 \%$ & slightly increased \\
\hline SP & 2.68 & 2.28 & & & & & $2.7-13.3$ & $3.6-15.6$ & $2.3-9.9$ & & \\
\hline $\mathrm{FC}(\%)$ & $21.52 *$ & $16.60^{*}$ & $9.6-19.23 \%$ & $50-84.30 \%$ & $53 \%$ & $4 \%$ & & & & ca. $39 \%$ & slightly increased \\
\hline FS & $\begin{array}{l}21.00^{30 \mathrm{~min}} \\
18.00^{60 \mathrm{~min}}\end{array}$ & $\begin{array}{l}26.00^{30 \mathrm{~min}} \\
21.00^{60 \mathrm{~min}}\end{array}$ & & & $10 \%$ & $9 \%$ & & & & ca. $60 \%$ & relatively similar \\
\hline viscosity $\left(\mathrm{Ns} / \mathrm{m}^{2}\right)$ & 17.52 & 22.18 & & & & & & & & & \\
\hline pbulk $\left(\mathrm{g} / \mathrm{cm}^{3}\right)$ & 1.25 & 0.94 & & & & & & & & $0.649 \pm 0.05$ & $0.649 \pm 0.05$ \\
\hline $\mathrm{pH}$ & 6.72 & 6.51 & & & & & & & & & \\
\hline $\mathrm{pI}$ & & & $4-5$ & & 4.5 & & & & & & \\
\hline solubility $(\mathrm{g} / \mathrm{g})$ & & & & & & & $21-143$ & $36-147$ & $52-200$ & & reduced \\
\hline $\mathrm{t}_{\min }$ cooking $(\mathrm{min})$ & & & & & & & & & & 22 & $14-21$ \\
\hline WUR & & & & & & & & & & $1.43 \pm 0.002$ & $1.45-1.56$ \\
\hline elongation ratio & & & & & & & & & & $1.26 \pm 0.08$ & $1.23-1.4$ \\
\hline GSL (\%) & & & & & & & & & & $20.43 \pm 0.006$ & $12.13-20.10$ \\
\hline$-\mathrm{L} / \mathrm{B}$ ratio & & & & & & & & & & $1.62 \pm 0.21$ & $1.59-1.62$ \\
\hline
\end{tabular}


a balance between low water consumption while processing the velvet bean also saving energy.

\section{POTENTIAL COMMERCIALIZATION OF VARIOUS PROCESSING}

Commercial velvet bean powder has been available to cure Parkinson's disease. This is under the protection of US Patent No. 7470441B2 and US7470441B2, WO2017126959A1, and EP1567177A2. The most commercialization of velvet bean use is in the form of a medicine regime. As far as it is concerned, the patent or other intellectual properties of instant velvet bean flour as a food ingredient to be raw materials of functional foods is not found. Another commercialization potential is the use of the flour as protein-lipid rich food ingredients for daily menu components. A holistic value chain from agriculture to end-users of velvet bean flour also considered as environmentally friendly food chain because the mucuna plant is excellent green manure, controlling weed in the soil through its allelochemicals, DOPA; capable of nitrogen fixation in the soil, a good protein-lipid balance provision comparative to soybean, and can optimally support food sovereignty by optimal use of unfertile soils.

The patent No. 7470441B2 is about an extraction method for $M$. pruriens seeds to produce fractions or crude extract that contain at least one of active compounds, substance, ot their mixtures for pharmaceutical uses capable of preventing, reducing, and curing Parkinson's disease. The successive method uses solvents comprised of hexane, acetone, water-ethanol which contains vitamin $\mathrm{C}$ at particular levels. Pharmaceutically, the extract gives broader therapy including LDOPA therapy, slowing down the development of the disease even though it onsets earlier yet the development can be retarded as well as to prevent or eliminate acute and chronic L-DOPA toxicity.

Patent application No. WO2017126959A1 is about food products containing fermented starch from diverse starch resources with claims of fermented starch content at $15 \%$ and inoculum levels of 0.1 $10 \%$ providing health benefits and the food products are organoleptically well-liked by consumers. The claim covers up the processing methods for commercial productions i.e. the uses of particular moisture content, heating, cooling for appropriate fermentative temperature, inoculation for the claimed method.

Patent application No. EP1567177A2 is pointing out $M$. pruriens for neuroligical disease treatments focusing on pharmaceutical compositions that containing $M$. pruriens seeds or one or more of its components, substances, fractions, as well as their mixtures to prevent, remove or to treat the diseases. The claims also include methods to prepare them from $M$. pruriens for the aforementioned goals. The uses of this product include a wider spectrum of Parkinson's disease from retarding the need to give combination treatments initial onset, and the time course of L-DOPA effication as well as to prevent or to eliminate acute or chronic L-DOPA treatments. The focused components are bipolarlipophilic fraction compounds either in the form of an infusion, injection solutions, gelatin capsule, tablets, and slow-released tablets. The targeted compounds under the claims have neurostimulator and neuroprotective functions obtained from particular M. pruriens (M-PL0100, M-EL100, MBL0100, LAT543-0 dan M-W-EL1299, M-W0100, MWEL0700, M-ML0100). The scope of pharmaceutical preparation composition is (1) to inhibit L-DOPA metabolism or dopamine, (b) to improve L-DOPA absorption to generate initial LDOPA effication and (c) to lengthen L-DOPA effication. Patent No. US7470441B2 claims extraction method of $M$. pruriens seeds using hexane, the mixture of water and ethanol containing $0.5 \%$ vitamin $\mathrm{C}$ for pharmaceutical goals.

Such pharmaceutical preparations can have environmental risks because of organic solvent waste. Moreover, the area where velvet beans produced is categorized by a fragile environment in sustainable viewpoints, which warrant another processing method that more environmentally friendly. Clean water is an obstacle in this area to make the velvet beans safe from high levels of LDOPA and cyanides. To be positive towards green manure contributions of velvet bean planting, thus the commercialization model is important to be integrated commercialization system. 
Commercialization at small-medium scale enterprises producing foods or food ingredients is considered as more realistic. Therefore, food processing oriented for health ingredients is important. Such food processing technology with less water consumption is investigated to achieve a system of food commercialization for velvet beans and oriented to get health-related food materials. It is expected that less water consumption processing would give better economic feasibility.

\section{CONCLUSION}

Velvet bean uses for health-related food product development warrant research on a more holistic approach to achieving sustainable commercialization in a way that environmentally friendly, by reducing the use of organic solvents, less water consumption during processing, while during its farming the mucuna plant could give healthier soils. Consequently, the healthier the soils, the better the agricultural productions and finally, and hopefully, society gets benefits for their well being and welfares.

Based on this literature reviews it is concluded that steaming might the best compromising of functional food ingredients where low water consumption, safe and effective energy uses because of the power of steam and latent energy involved. The expectation is capable of producing safe functional food ingredients for diabetes mellitus and/or Parkinson by the use of L-DOPA, cyclic-D-chiro inositol, and fibers also of the basic nutrients. The protein content of velvet bean comparable to that of soybean yet growth values (protein effective ratio) is poor so monotonous diet based velvet beans is not recommended. As functional ingredient, it can be managed to reach safe dosages in daily intakes. However, all hypotheses were currently being proven in our research group. Using steaming and germinating to characterizing steamed-whole flour velvet beans.

\section{REFERENCES}

Adebayo-Oyetoro, A.O., Olatidoye, O.P., Ogundipe, O.O., Balogun, O.I. and Faboya, A.O. 2011. Quality evaluation of weaning food produced from blend of ofada rice (Oryza sativa) and bambara groundnut (Voandzeia subterranean l.). Electronic Journal of Environmental, Agricultural and Food Chemistry. 10(6), pp.2322-2330.

Adebowale, K.O. and Lawal, O.S. 2003. Microstructure, physicochemical properties and retrogradation behaviour of Mucuna bean (Mucuna pruriens) starch on heat moisture treatments. Food Hydrocolloids. 17(3), pp.265272 DOI: $10.1016 / \mathrm{S}) 268-005 \mathrm{X}(0.2) 00076-0$

Adebowale, Y.A., Adeyemi, A. and Oshodi, A.A. 2005. Variability in the physicochemical and antinutritional attributes of six Mucuna species, Food Chemistry. 89, pp.37-48.

Afolabi, O.A., Oshuntogun, B.A., Adewusi, S.R., Fapojuwo, O.O., Ayorinde, F.O., Grisson, F.E. and Oke, O.L. 1985. Preliminary nutritional and chemical evaluation of raw seeds from Mucuna solanei: An underutilized food source. Journal of Agricultural Food Chemistry. 33, pp.122-124.

Agbede, J.O. and Aletor, V.A. 2005. Studies of the chemical composition and protein quality evaluation of differently processed Canavalia ensiformis and Mucuna pruriens seed flours. Journal of Food Composition and Analysis. 18(1), pp.89-103.

Ahenkora, K., Dadzie, M. and Osei-Bonzu, P. 1999. Composition and functional properties of raw and heat processed velvet bean flours. International Journal of Food Science and Technology. 34(2), pp.131-135.

Ajayi, I.A., Oderinde, R.A., Kajogbola, D.O. and Ukponi, J.U. 2006. Oil content and fatty acid composition of some underutilized legumes from Nigeria. Food Chemistry. 99, pp.115-120.

Balogun, I.O. and Olatidoye, O.P. 2012. Chemical composition and nutritional evaluation of velvet bean seeds (Mucuna utilis) for domestic consumption and industrial utilization in Nigeria. Pakistan Journal of Nutrition. 11(2), pp.116-122. ISSN 1680-5194

Balogun, I.O. and Olatidoye, O.P. 2010. Functional properties of dehulled and undehulled velvet 
beans flour (Mucuna utilis). Journal of Biological Sciences and Bioconservation. 2, pp.1-10.

Balogun, O.I., Epriliati, I., Otunola, E.T. 2015. Potential useof species Rhizopus for removal of L-DOPA from Mucuna utilis (Wight). Presented at $8^{\text {th }}$ HerbFest Symposium Food as medicine: utilization and sustainable exploitation of African Medicinal Plants and natural Products. Abuja, Nigeria 6-8 October, 2015.

Balogun, O.I., Epriliati, I., Otunola, E.T. and Agboola, H.A. 2017. Rhizopus oryzae FNCC 6010, Rhizopus oligosporus FNCC 6011, and their hybrid lowered antioxidant capacity of velvet beans compared to germination. International Food Research Journal. 24(4), pp.1606-1613.

Bhat, R., Sridhar, K.R., Young, C-C., Arun, A., Bhagwath, A.A. and Ganesh, S. 2008. Composition and functional properties of raw and electron beam-irradiated Mucuna pruriens seeds. International Journal of Food Science and Technology.43, pp.1338-1351.

Bhat, R. and Karim, A.A. 2009. Exploring the nutritional potential of wild and underutilized legumes. Comprehensive Reviews in Food Science and Food Safety, 8(4), pp.305-331.

Bhat, R., Sridhar, K.R. and Tomita-Yokotani, K. 2007a. Effect of ionizing radiation on antinutritional features of velvet bean seeds (Mucuna pruriens). Food Chemistry. 103, pp.860-866.

Bhat, R., Sridhar, K.R., and Velmourougane. 2007b. Microbial quality evaluation of velvet bean seeds (Mucuna pruriens L. DC.) exposed to ionizing radiation. Tropical and Subtropical Agrosystems. 7, pp.29-40.

Bressani, R. 2002. Factors influencing nutritive value in food grain legumes; Mucuna in comparison to other grain legumes. In: Flores (eds) Mucuna as a food and feed: current uses and the way forward. proceedings of an international workshop hold Apr 26-29, 2000 in Tegucigalpa, Honduras pp,164-188
Bressani, R., Lau, M. and Silvia Vargas, M. 2003. Protein and cooking quality and residual content of dehydroxyphenylalanine and of trypsin inhibitors of processed Mucuna beans (Mucuna spp). Tropical and Subtropical Agroecosystemsi. 1, pp.197-212.

Buckles, D. 1995. Velvet bean: A "new" plant with a history. Economic Botany. 49, pp.13-25.

Capo-chichi, L.J., Eilitta, M., Carsky, R.J., Gilbert, R.A. and Maasdorp, B. 2003. Effect of genotype and environment on L-dopa concentration in Mucuna's (Mucuna sp.) seeds. Tropical and Subtropical Agroecosystems. 1, pp.319-328.

Dabija, A. 2012. A study establishing an optimum malting diagram. Food and Environment Safety-Journal of Faculty of Food Engineering, Stefan cel MareUniversitySuceava. XI(2), pp.85-90.

Diallo, O.K., Kante, S., Myhrman, R., Soumah, M., Cisse, N.Y. and Berhe, T. 2002. Increasing farmer adoption of Mucuna pruriens as human food and animal feed in the Republic of Guinea. In Flores, B.M., Eilitta, M., Myhrman, R., Carew, L.B. and Carsky, R.J., Eds. Whorkshop, Cidicco, CIEPCA and World Hunger Research Center, Tegucipalga, Honduras (April 26-29, 200), pp.60-72 http://www.cidicco.hn/newcidiccoenglish/Muc una\% 20book $/ 2$.Diallo20\%et $20 \%$ al $20 \%$.pdf

Diallo, O.K. and Berhe, T. 2003. Processing of Mucuna for human food in the Republic of Guinea. Tropical and Subtropical Agroecosystems. 1, pp.193-196.

Divya, B.J., Suman, B., Venkataswamy, M., and Thyagaraju, K. 2017. The traditional uses and pharmacological activities of Mucuna pruriens (L.)DC: a comprehensive review. Indo American Journal of Pharmaceutical Research, 7, (01), 7516-7525.

Dossa, C.S., Mensah, G.A., Dossa, A.D. and Adoun, C. 1998. Influence of various physicochemical treatments of Mucuna 
pruriens seeds on the nutrient chemical composition. Tropiculture. 16-17, pp.141.

Dossa, E.L., Fernandez, E.C.M. and Reid, W.S. 2008. Above and belowground biomass, nutrient and carbon stocks contrasting an opengrown and a shaded coffee plantation. Agroforestry Systems. 72, pp.103-115.

Egounlety, M. 2003. Processing of velvet bean (Mucuna pruriens var utilis) by fermentation. Tropical and Subtropical Agroecosystems. 1, pp.173-181.

Elvinger, F., Akey, B.L., Senne, D.A., Pierson, F.W., Porter-Spalding, B.A., Spackman, E. and Suarez, D.L. 2007. Characteristic of diagnostic tests used in the 2002 low pathogenic avian influenza H7N2 outbreak in Virginia. Journal of Veterinary Diagnosis Investigation. 19(4), pp.341-348.

Eucharia, N.O. and Edward, O.A. 2010. Allelopathy as expressed by Mucuna pruriens and the possibility for weed management. International Journal of Plant Physiology and Biochemistry. 2(1), pp.1-5.

Ezeagu, I.E., Maziya-Dixon, B. and Tarawali, G. 2003. Seed characteristics and nutrient and antinutrient composition of 12 Mucuna accessions from Nigeria. Tropical and Subtropical Agroecosystems. 1, pp.129-140.

Ezueh, M.I. 1997. Cultivation and utilization of minor food legumes in Nigeria. Tropical grain Legumes Bulletin, 10, International Institute for Tropical Agriculture, Ibadan, Nigeria

Ferreira, J.F.S., Simon, J.E., and Janick, J. 1995. Developmental Studies of Artemisia annua : Flowering and Artemisinin Production Under Greenhouse and Field Conditions. Planta Medica. 61, pp.167-170.

Ferreira, J.F. and Janick, J. 2004. Allelopathic plants. XVI. Artemisia species. Allelopathy Journal. 14(2), pp.167-175.

Fujii, Y. 2003. Allelopathy in the natural and agricultural ecosystems and isolation of potent allelochemicals from velvet bean (Mucuna pruriens) and hairy vetch (Vicia villosa). Biology Science Space. 17, pp.6-13 doi: $10.2187 /$ bss. 17.6

Garcia-Echeverria, C.L. and Bressani, R. 2006. Effect of different cooking treatments of Mucuna beans on its L-Dopa content. Archivos Latinoamericanos de Nutricion. 56, pp.175184. http://www.alanrevista.org/ediciones/ 2006-2/coccion_frijol_mucuna.asp

Gardjito, M., Djuwardi, A., and Harmayani, E. 2013. Pangan Nusantara karakteristik dan prospek untuk percepatan diversifikasi pangan. $1^{\text {st }}$ ed. Jakarta: Kencana Prenada Media Group.

Gilbert, R. 2002. Mucuna pruriens in Malawi: A promising legume with a troubled history. In Flores, B.M., Eilitta, M., Myhrman, R., Carew, L.B. and Carsky, R.J., Eds. Workshop, cidicco, CIEPCA and World Hunger Research Center, Tegucipalga, Honduras (April 26-29, 200), pp.18-47.

http://www.cidicco.hn/newcidiccoenglish/Muc una\%20book/c.CIDICCO.pdf

Giuliano, F. and Allard, J. 2001. Dopamine and male sexual function. European Urology. 40, 601-608

Golisz, A., Sugano, M., Hiradate, S., and Fujii, Y. 2011. Microarray analysis of Arabidopsis plants in response to allelochemical L-DOPA. Planta. 233, pp.231-40 doi: 10.1007/s00425010-1294-7

Gurumoorthi, P. and Uma, S. 2011. Heat-stable and heat-labile antinutritional profile in Mucuna pruriens var utilis: effected by germination. International Food Research. 18(4), pp.14211426

Gurumoorthi, P., Janardhanan, K. and Myhrman, R.V. 2008. Effect of differential processing methods on L-DOPA and protein quality in velvet bean, an underutilized pulse. $L W T, 41$, pp.588-96. 
Harmayani, E., Santosa, U., and Gardjito, M. 2016. Makanan Tradisional Indonesia: Kelompok makanan fermentasi dan makanan popular di masyarakat. Yogyakarta: Gadjah mada Press. pp.13-18.

Heyne, K. 1987. Tumbuhan berguna Indonesia. Vol I and II. Badan Litbang Kehutanan (Translator). $1^{\text {st }}$ ed. Jakarta Pusat: Koperasi karyawan Departemen Kehutanan

Higasa, S., Negishi, Y., and Sugahara, T. 1996. Changes in free amino acids of tempe during preparation with velvet beans (Mucuna pruriens). Journal of the Japanese Society for Food Science and Technology-Nippon Shokuhin Kagaku Kogaku Kaishi. 43, pp.188193.

Iauk, L., Galati, E.M., Forestieri, A.M., Kirjavainen, S. and Trovoato, A. 1989. Mucuna pruriens decoction lowers cholesterol and total lipid plasma levels in the rat. Phytoteraphy Research. 3, pp.263-264

Infante, M.E., Perez, A.M., Simao, M.R., Manda, F., Baquete, E.F., Fernabdes, A.M. and Cliff, G.L. 1990. Outbreak of acute toxic psychosis attributed to Mucuna pruriens. The Lancet, 336 ,

pp.1129. https://www.jstage.jst.go.jp/article/nskkk1995/ 43/2/43_2_188/_article

Ingalhalikar, S., Page, N., Gaikwad, S. and Gurav, R.V. 2017. Mucuna laticifera, a new species from north-eastern India. Phytotaxa. 319(1), pp.118-122

https://doi.org/10.11646/phytotaxa.319.1.8 http://www.maprese.com/j/pt/

Kakade, M.L. and Evans, R.J. 1965. Growth inhibition of rats fed navy bean fractions. Journal of Agricultural Food Chemistry, 13, pp.470

Kala, B.K. and Mohan, V.R. 2012. Effect of microwave treatment on the antinutritional factors of two accessions of velvet bean, Mucuna pruriens (L.) DC. var. utilis (Wall. ex Wight) bak. ex Burck. International Food Research Journal. 19(3), pp.961-969
Katzenschlager, R., Evans, A., and Manson, A. 2004. Mucuna pruriens in Parkinson's disease: a double blind clinical and pharmacological study. Journal of Neurology Neurosurgery and Psychiatry. 75, pp.1672-1677.

Liener, I.E. and Kakade, M.L. 1980. Protease inhibitors. In: Liener, I.E. ed. Toxic Constituents of Plant Foodstuffs. $2^{\text {nd }}$ Ed. New York: Academic Press. Inc., pp.7-71.

Lorenzetti, B.B., Souza, G.E., Sarti, S.J., Santos Filho, D. and Ferreira, S.H. 1991. Myrcene mimics the peripheral analgesic activity of lemongrass tea. Journal of Ethnopharmacology. 34, pp.43-48.

Lorenzetti, E., MacIsaac, S., Arnason, J.T., Awang, D.V.C. and Buckles, D. 1998. The phytochemistry, toxicology and food potential of velvet bean (Mucuna adans spp. Fabaceae). In D. Buckles, O. Osiname, M. Galiba, and G. Galiano, Cover crops of West Africa; contributing to sustainable agriculture. IDRC, Ottawa, Canada and IITA, Ibadan, Nigeria, pp. 57

Marimuthu, M. and Gurumoorthi, P. 2013. Physicochemical and functional properties of starches from Indian Jack bean (Canavalia ensiformis), an underutilized wild food legume. Journal of Chemical and Pharmaceutical Research. 5(1), pp.221-225.

Mary-Josephine, R. and Janardhanan, K. 1992. Studies on chemical composition and antinutritional factors in three germplasm seed materials of the tribal pulse, Mucuna pruriens (L.) DC. Food Chemistry. 43, pp.13-18.

Mohan, V.R. and Janardhanan, K. 1995. Chemical analysis and nutritional assessment of lesser known pulses of the genus Mucuna. Food Chemistry, 52, pp.275-280.

Montgomery, R.D. 1980. Cyanogen. In Liener, I.E. ed. Toxic constituents of plant food stuffs New York: Acadmic Press, pp.158-160.

Mugendi, J.B. and Njagi, E.M. 2010. Effects of processing mucuna bean (Mucuna pruriens L.) 
on protein quality and antinutrients content. Conference on International Research on Food Security, natural Resources management and Rural Development, ETH Zurich, Sept 14-16, 2010 .

Mugendi, J.B.W., Njagi, E.N.M., Kuria, E.N., Mwasaru, M.A., Mureithi, J.G. and Apostolides, Z. 2010a. Nutritional quality and physicochemical properties of Mucuna bean (Mucuna pruriens L.) protein isolates. International Food Research Journal. 17, pp.357-366.

Mugendi, J.B.W., Njagi E.N.M., Kuria, E.N., Mwasaru, M.A. and Mureithi, J.G. 2010b. Effects of processing technique on the nutritional composition and anti-nutrient content of mucuna bean (Mucuna pruriens L.). African Journal of Food Science. 4(4), pp.156166.

Mugendi JB and Njagi EM. 2010. Effects of processing mucuna bean (Mucuna pruriens L.) on protein quality and antinutrients content. Conference on International Research on Food Security, natural Resources management and Rural Development, ETH Zurich, Sept 14-16, 2010 .

Mwangi, H.W., Mureithi, J.G. and Gachene, C.K.K. 2006. Legume cover crops for conservation agriculture in arid and semi arid regions of Machakos District, Eastern Province. Legume Research Network Project Newsletter. 12, pp.2-4.

Nwaichi, E.O., Wegwu, M.O. and Onyeike, E.N. 2009. Phytoextracting cadmium and copper using Mucuna pruriens. African Journal of Plant Science. 3(12), pp.277-282.

Nyirenda, D., Musukwa, M. and Jonsson, L.O. 2003. The effect of different processing methods of velvet bean (Mucuna pruriens) on L-dopa content, proximate compositionand broiler chicken performance. Tropical and Subtropical Agroecosystems. 1, pp.253-260.

Osei-Bonsu, P., Hossain, M. and Soza, R.F. 1994. Potential uses of Mucuna as legume crop on
Ghana. National Workshop on Food, Kumani Institute, Ghana

Onweluzo, J.C., Obanu, Z.A. Okwandu, M.C. 2004. Potential of gum from Detarium microcarpum (DM) and Mucuna flagellipes (MF) seeds as raw beef burger stabilizers. Plant Foods Human Nutrition. 59, pp.137-141.

Onwuka, G. 1997. Toxicology studies of hemagglutinin in soy bean and Mucuna cochinchinensis and growth response of Clarias gariepinus. $\mathrm{PhD}$ Thesis, University of Nigeria, Nsukka, Nigeria

Patent US No. 7470441B2

Patent US7470441B2

Patent WO2017126959A1

Patent EP1567177A2

Patent No. 7470441B2 . US7470441B2

Patent WO2017126959A1

Patent EP1567177A2

Pierson, J., Norris, J.L., Aerni, H-R., Svenningsson, P., Caprioli, R.M. and Andren, P.E. 2004. Molecular profiling of experimental parkinson's disease: direct analysis of peptides and proteins on brain tissue sections by Maldi Mass spectrometry. Journal of Proteomic Research. 3, pp.289-295.

Phytochemical and Ethnobotanical Databases at Phytochemical Database, USDA-ARS-NGRL, Beltsville Agricultural Research Center, Beltsville, Md.

Prakash, U., Bhuvameswari, S., Balamurugan, A., Karthik, A., Deepa, S., Aishwarya, H., Manasveni, and Sahana, S. 2013. Studies on bio activity and phytochemistry of leaves of common trees. International Journal of Research in Pharmaceutical Sciences. 4(3), pp.476-481.

Prakash, D. and Tewari, S.K. 1999. Variation on LDOPA content in Mucuna species. Journal of 
Medicinal and Aromatic Plant Science. 21, pp.343-346

Pramono, I.B. 2010. Peningkatan produktivitas lahan miring berbahan induk kapur dengan sistem agroforestri untuk ketahanan pangan dan pengendalian lahan erosi. Laporan Hasil Penelitian Program Insentif Riset Terapan. Kementerian Kehutanan.

Rajaram, N. and Janardhanan, K. 1991. The biochemical composition and nutritional potential of the tribal pulse Mucuna gigantea Wild DC. Plant Foods for Human Nutrition. 41, pp.45-52.

Raval, K., Vaswani, P.S. and Majumder, D.R. 2012. Biotransformation of single amino acid L-tyrosine into a bioactive molecule L-DOPA. International Journal of Scientific and Research Publications. 2(5), pp.1-9.

Ravindran, V. and Ravindran, G. 1988. Nutritional and antinutritional characteristics of Mucuna bean seeds. Journal of Science of Food and Agriculture. 46, pp.71-79.

Rodgers, K.J., Hume, P.M., Dunlop, R.A. and Dean, R.T. 2004. Biosynthesis and turnover of DOPA-containing proteins by human cells. Free Radical Biology Medicine. 37, pp.17561764. doi: 10.1016/j.freeradbiomed.2004.08.009

Saha, H.M. and Muli, M.B. 2000. What the coastal farmer sees in Mucuna? Legume Research Network Project Newsletter, KARI, 3, pp. 15.

Sandberg, A.S. 2002. In vitro and in vivo degradation of phytate. In Reddy, N.R. and Sathe, S.K., Eds. Food Phytates. Boca Raton, Florida: CRC Press, pp.139-155.

Sardjono, S.D. 1995. Analgesik Opioid dan Antagonis, dalam Farmakologi dan Terapi, $4^{\text {th }}$ Ed., Jakarta: Fakultas Kedokteran-Universitas Indonesia.

Sardjono, R.E., Musthapa, I., Khoerunisa, F., Qowiyah, A., and Rachmawati, R. subchronic toxicity of ethanolic extract velvet bean
(Mucuna pruriens) from Indonsia.Pertanika Journal Tropical Agricultural Sciences, 41(3), pp.1497-1509.

Sardjono, R.E., Musthapa, I., Sholihin, H. and Ramdhani, R.P. 2012. Physicochemical composition of Indonesian velvet bean (Mucuna pruriens L.). Global Journal of Research on Medicinal Plants and Indigenous Medicine, 1(4), pp.101-108.

Seena, S. and Sridhar, K.R. 2005. Physicochemical, functional and cooking properties of under explored legumes, Canavalia cathartica Thouars and C. maritima Thouars of the southwest coast of India. Current Science. 90, pp.1638-1650.

Soares, A.R., Marchiosi, R., de Cassia Siqueriasoares, R., de Lima, B.R., dos Santos, W.D. and Ferrarese-Filho, O. 2014. The role of LDOPA in plants. Plant Signal Behavior. 9, pp.e28275

http://www.scielo.br/scielo.php?pid=S1516184 62014000300732andscript=sci_arttextandtln $\mathrm{g}=\mathrm{es}$

Shaw, B.P. and Bera, C.H. 1993. A preliminary clinical study to cultivate the effect of vogorexSF in sexual disability patients. Indian Journal of Internal Medicine. 3, 165-169.

Siddhuraju, P., Vijayakumari, K. and Janardhanan, K. 1996. Chemical composition and protein quality of the little-known legume, velvet bean (Mucuna pruriens (L.) DC). Journal of Agricultural Food Chemistry. 44, pp.26362641.

Siddhuraju, P. and Becker, K. 2001. Effect of various domestic processing methods on antinutrients and in vivo protein and starch digestibility of two indigenous varieties of Indian tribal pulse, Mucuna pruriens var. utilis. Journal of Agricultural Food Chemistry. 49, pp.3058-3067.

Siddhuraju, P., Becker, K. and Makkar, H.P.S. 2000. Studies on the nutritional composition and antinutritional factors of three different germplasm seed materials of an underutilized 
tropical legume. Mucuna pruriens var. utilis. Journal of Agricultural Food Chemistry. 48, pp.6048-6060.

Sridhar, K.R. and Bhat, R. 2007. Agrobotanical, nutritional and bioactive potential of unconventional legume - Mucuna. Livestock Research for Rural Development. 19(9), pp.134.

Sudiyono. 2010. Penggunaan $\mathrm{Na}_{2} \mathrm{HCO}_{3}$ Untuk Mengurangi Kandungan Asam Sianida ( $\mathrm{HCN})$ Koro Benguk Pada Pembuatan Koro Benguk Goreng. Agrika. 4(1), pp.48-53.

Topa, S. and Kocacaliskan, I. 2006. Allelopathic effects of DOPA against four weed species. DPU Fen Bilimleri Enstitusu. 11, pp.27-32.

Tripathi, Y.B. and Upadhyay, A.K. 2001. Antioxidant Property of Mucuna pruriens Linn. Current Science. 80(11), pp.1377-1378.

Tuleun, C.D., Patrick, J.P. and Tiamiyu, L.O. 2009. Evaluation of raw and boiled velvet bean (Mucuna utilis) as feed ingredient for broiler chickens. Pakistan Journal of Nutrition. 8(5), pp.601-606.

Udedibie, A.B.I. and Carlini, C.R. 1998. Brazilian Mucuna pruriens seeds (velvet beans) lack haemagglutinating activity. Journal of Agricultural Food Chemistry. 46, pp.14501452.

Ukachukwu, S.N. and Obioha, F.C. 2000. Effect of time duration of thermal treatments on the nutritive value of Mucuna cochinchinensis. Global Journal of Pure and Applied Science, 6, pp.11-15.

Vadivel, V. and Bielsaski, H.K 2012. Effect of certain indigenous processing methods on the bioactive compounds of ten different wild type legume grains. Journal of Foodscienceand Technology, 49(6), pp.673-683.

Vadivel, V. and Janardhanan, K. 2000. Nutritional and antinutritional composition of velvet bean: an under-utilized food legume in South India.
International Journal of Food Science and Nutrition. 51, pp.279-287.

Versteeg, M.N., Amadaji, F., Eteka, A., Houndekon, V. and Manyong, V.M. 1998. Collaboration to increase the use of Mucuna in production systems in Benin. In Buckles, D., Eteka, A., Osiname, O., Galiba, M. and Galiano, G. Eds. Cover crops in West Africa: contributing to sustainable agriculture. IDRC, IITA and SG2000, Ottawa, Canada, pp.33-34.

Vijayakumari, K., Siddhuraju, P. and Janardhanan, K. 1996. Effect of different post-harvest treatments on antinutritional factors in seeds of the tribal pulse, Mucuna pruriens (L.) DC. International Journal of Food Science and Nutrition, 47, pp.263-272.

Wanita, Y.P. and Rahayu, S. 2011. Effects of tempeh's types, blanching and skim addition on consumer acceptance of nut tempeh yoghurt. Proceeding The $3^{\text {rd }}$ International Conference of Indonesian society for lactic acid bacteria.

Wanjekeche, E., Wakasa, V., and Mureithi, J.G. 2003. Effect of germination, alkaline and acid soaking and boiling on the nutritional value of mature and immature mucuna (Mucuna pruriens) beans. Tropical and Subtropical Agroecosystems. 1, pp.183-192.

Winarni, S., Judiwati, R., Prajogo, B., and Hayati, A. 2011. Fraksi etanol $96 \%$ biji koro benguk (Mucuna pruriens L.) sebagai peningkat kualitas spermatozoa mencit (Mus musculus), Jurnal Kesehatan Reproduksi, 1, 60-66.

Zijlmans, J.C.M, Katzenschlager, R., Daniel, S.E., and Lees, A.J.L. 2004. The L-dopa response in vascular parkinsonism. Journal of Neurology, Neurosurgery and Psychiatry. 75, pp.545-547. 